\title{
ASM-ROBOT: A Cyber-Physical Home Automation Controller with Memristive Reconfigurable State Machine
}

\author{
Kennedy Chinedu Okafor ${ }^{1}$, Omowunmi Mary Longe ${ }^{2}$ \\ Dept. of Mechatronics Engineering, Federal University of Technology, Owerri, Nigeria ${ }^{1}$ \\ Electrical and Electronic Engineering Science, University of Johannesburg, South Africa ${ }^{1,2}$
}

\begin{abstract}
In the next 5 to 10 years, digital Artificial Intelligence with Machine Circuit Learning Algorithms (MCLA) will become the mainstream in complex automated robots. Its power concerns, ethical perspectives, including the issues of digital sensing, actuation, mobility, efficient processcomputation, and wireless communication will require advanced neuromorphic process variable controls. Existing home automated robots lack memristic associative memory. This work presents Cyber-Physical Home Automation System (CPHAS) using Memristive Reconfigurable Algorithmic State Machine (MRASM) chart. A process control architecture that supports Concurrent Wireless Data Streams and Power-Transfer (CWDSPT) is developed. Unlike legacy systems with powersplitting (PS) and time-switching (TS) controls, the MRASMROBOT explores granular wireless signal controls through unmodulated high-power continuous wave $(\mathrm{CW})$. This transmits infinite process variables using Orthogonal Space-Time Block Code (OSTBC) for interference reduction. The CWDSPT transmitter and receiver circuit design for signal processing are implemented with complexity noise-error reduction during telemetry data decoding. Received signals are error-buffered while gathering control variables' status. Transceiver Memristive neuromorphic circuits are introduced for computational acceleration in the design. Hardware circuit design is tested for system reliability considering the derived schematic models for all process variables. Under small range space diversity, the system demonstrated significant memory stabilization at the synchronous iteration of the synaptic circuitry.
\end{abstract}

Keywords-Cloud computing; cyber-physical systems; complex robot; computational science; IoT; machine learning

\section{INTRODUCTION}

\section{A. Background}

Memristive Reconfigurable Algorithmic State Machine Robot (MRASM-ROBOT) is a novel intelligent automation approach that provides support for the control of converged appliances using localized space diversity. The concept is very useful for providing security to both localized and remote homeowners. It exploits associative memory which has a structured memory unit for storing identified data both at the edge and cloud control levels respectively. The system uses access by data content (content addressable memory), and address by memory location (associative memory).

Memristive neuromorphic circuits can be constructed with associative memory for infinite process variables such as temperature, smoke, flames, gases, humidity, and water, among others. Modern-day hardware modules can easily be implemented using Memristive reconfigurable memory systems. This is found in neuromorphic robots and other complex systems [1]. The application areas are very huge, thereby creating demands for location-independent automation processes. Smart Internet of Things (IoT) is often used in this regard due to space diversity considerations. In such cases, IoT RF technology (such as ATMEL Smart-SAM25 module) with the CWDSPT technique can be adapted to provide effective monitoring and control of both on-premises and remote processes. With heightened security challenges as well as domestic havoc at homes and offices, such an automated security system becomes very handy [1], [2].

Existing home automation approaches fail to account for space diversity sensitivity. Also, process variable coordination in various application contexts is unpredictable. In most legacy systems, their efficiency often involves a high cost, especially when using remote network services. There is a need for an automated security system that is optimized for high efficiency in controlling home appliances at short-range communication. Smart Energy Audit Systems (SEACS) have been proposed which largely depends on IoTs via near field communication (NFC) [2]. Hand-gesture-based control Robots have been implemented for real-time interactive control systems targeted at household appliances [3]. Home automation systems for anomaly classification using unsupervised probabilistic associations for sensing and event tracing in smart homes are trending [4], [5], and [6].

Recently, researchers are making efforts to drive biological neural networks and map with memristive neuromorphic models to customize AI-based Robots [7], [8], [9]. Similarly, memristive neuromorphic models have been applied in most computing acceleration designs [10], [11], [12]. In classical literature such as social psychology, the capacity to learn and recall the relationship-map existing among unconnected variables is referred to as associative memory [13]. This could be declarative in its structure or episodical [14] in its application contexts.

Various attributes of complex radio frequency (RF) robots such as real-time withdrawal reflex, classical eyeblink, etc., can occur via a complex learning process in associative memory. In this case, a conditioned reflex can be established between conditioned stimulus (CS) and system state-response. 
Low range RF memristive neuromorphic circuits can be leveraged as a Pavlov associative memory (PAM) or a withdrawal reflex [15], [16]. Max-input-feedback adaptivelearning rule has been applied to train the neuromorphic circuit while adapting it to PAM [17]. Some designs explore microcontrollers, and signal conditioners such as A/D converter to synthesize memristor features in a robot. In this case, the memristor can be used as a synaptic/ neuromorphic circuit, to model PAM [18]. Though finite state machine is not new, however, it can be employed to assist an unsupervised $k$ means logic intelligence while monitoring and detecting abnormal conditions.

\section{B. Advantages of Memristive Control Design Technique}

- Offers bifurcation analysis involving two or more parameters and predict the linear stability boundaries.

- Similar to biological synapses, dendrites, and neurons, the memristive technique with neuromorphic computing offers another layer to AI at a physical level.

- The memristive method offers insights into non-linear systems by extending the capabilities of resistors, Capacitors, and Inductors.

- The Memristive approach provides optimal control in circuit diversity.

Existing works on memristive systems focused on highdensity filters or FPGAs volatile memory, crossbar latches, neural networks, modeling of neural synapses, nonlinear oscillators, and filters. Very little work has been done field of Memristive reconfigurable state machine and space diversity control signaling.

\section{Research Contributions}

This work presents a novel control strategy for process variable transceiver modules in smart homes designs. The system allows for the optimization of errorless information decoding. The other contributions in this research include:

- Derive a standardized remote-controlled automation architecture adapted for process aggregation dynamics.

- To evaluate the IoT-gateway transmission and receiver behavior for a low bit error rate under the influence of Multipath Rayleigh Channel Space Vector (MRCSV) and White Gaussian noise (AWGN).

- To show the impact of space diversity on received signal using optimal OSTBC-combiner block (CB).

- To demonstrate MRASM-ROBOT use case scenario for bit error response at scale.

The rest of the paper is organized as follows. Section II presents related works. Section III presents the methodology. Section IV presents the smart automation engine (SAE). Section V presents the feedback scheduling algorithm, Section VI focused on space diversity control. Section VII discussed the experimental results, while Section VIII concludes the work.

\section{RELATED WORK}

Mostly, there are challenges regarding the coordination of control states and the collection of telemetry data for smart home analytics. The absence of MRASM-CWDSPT affects the accuracy of data communication on the home server. This leads to under-utilization and lower efficiency. In this section, closely related literatures on two important elements were studied namely: CWDSPT Space diversity and automated/smart homes without neuromorphic circuits.

\section{A. CWDSPT Space Diversity}

Concurrent Wireless Data Streams and Power-Transfer is an emerging area with great prospects especially with 5G rollout in Africa. In most works, spatial diversity (SD) is introduced in transmissions systems to mitigate the effect of multipath fading [19]. In lengthy links over extremely reflective surfaces like water bodies where a non-diversity link can't deliver high availability, SD is needed. It is used in multiple antennas that have similar characteristics involving real physical separation from each other. There have been several works on space diversity, but the work in [20] used a concave-convex procedure (CCCP) scheme to maximize the minimum rate of IoT nodes' private streams. This was done via the allocation of transmit power and adjustment of powersplitting ratios at the IoT edge nodes.

A similar work [21], discussed spatially modulated spacetime block code (HRSM-STBC) developed for two active antennas. To optimize space diversity schemes, extensive discussions on wireless power transfer (WPT) capabilities were presented in [22]. The work [23] investigated a wireless power transfer (DWPT) tool for developing wireless power transfer (WPT) systems and its related parameters. In [24], the authors proposed a radial-flux rotational wireless power transfer (RFR-WPT) system with a rotor state identification function for devices mounted on a rotating shaft. In [25], the authors focused on the systematic review of metamaterials and meta surfaces for wireless power transfer (WPT) and wireless energy harvesting (WEH) [26].

In [27], the authors leveraged Wireless power transfer (WPT) systems due to their security, convenience, and flexibility to propose a control strategy referred to as periodic energy control (PEC) in WPT. So far, current literature on wireless information power transfer and space diversity has not been explored in Cyber-physical home automation. Its applications in MRASM-ROBOT will be very novel and useful too.

\section{B. Neuromorphic State Machine (NSM)}

The authors [28], developed an inverter-based memristive neuromorphic RF switch on the microstrip line with good performance up to $5 \mathrm{GHz}$. Their design explored conductive bridging random access memory (CBRAM) scheme in which the switch uses metal-insulator-metal (MIM) structure, Copper/Nafion/Au majorly. In [29], the authors focused on inverter-based memristor crossbar neural networks. The work [30] investigated the impacts associated with the computing accuracy of analog memristive circuits for neuromorphic applications. In [31], the security application of memristive crossbar physical unclonable function was investigated for 
resource optimization. The work [32] investigated neuromorphic computational system models using the spine technique. This is used to improve system lifetime in mapping machine learning workloads. The work in [33] focused on the optimization of Memristive Crossbar Arrays. In [34], the authors investigated memristance variations capture synaptic weight variations. The authors [35], [36] proposed the Pavlov associative memory process otherwise known as multifunctional memristive Pavlov associative memory circuit. The work [37] proposed a weight optimization scheme that combines quantization and Bayesian inference for memristorbased neuromorphic computing system (NCS).

In cases of Mixed-signal analog/digital neuromorphic circuits, there has been the characterization of such circuits using ultra-low power consumption, real-time processing abilities, and low-latency response times [38]. In this case, neuromorphic processors are used as the neuromorphic agent. The work [39] discussed a new neuro-inspired, hardwarefriendly readout stage for the liquid state machine (LSM) using neuromorphic VLSI implementation. The work [40] discussed a neuromorphic computational scheme known as the prefrontal cortex (PFC). The work is equally looked at as mixed-signal analog/digital neuromorphic implementation. Authors [41] highlighted that memristive circuits can trigger the associative memory processes while retaining the design parameters.

There are other types of memristive circuits without any clear synaptic and neuron circuits. Hence, with a modified memristive circuit, the memristive neuromorphic models can be employed to enhance computational efficiency in deployment contexts.

Considering the memristive neuromorphic circuits used for computation acceleration, the work [42], [43], [44], showed that the synaptic circuit is required to represent process variable weights in binary form. The works equally highlighted that various synaptic circuits can be designed to utilize neuromorphic circuits. At the implementation level, Field programmable gate arrays (FPGAs) have been used to provide neuromorphic reconfigurable characteristics while enabling flexible schematic designs for various applications [44], [45], [46], [47]. Interestingly, various control circuit topologies designated for process variables in existing designs lack the functionality of MRASM states. Also, the design of memristive neuromorphic circuits must replicate the process of learning, with high retention for conditional state-based signals.

Therefore, the gap in NSM is that low-end cyber-physical applications cannot fit into the design complexity due the memory stabilization issues. However, the use of associative memory can help in the design of Cyberphyscal robots that learns and remembers the relationship between unrelated things. Such reconfigurable NSM can be useful in the design of intelligent RF systems.

\section{Summary of Related Works (Automated Home)}

The use of NSM to drive space diversity intelligent systems is novel. In this section, the summary current related efforts on automated/smart home without NSM. In [48], the authors proposed Open-source home automation systems which lacked a detailed implementation framework. In the work [49], the authors proposed home energy management systems (HEMS). The design supports end-users by allowing for demand-side management and automation. In [50], the authors developed a secured but lightweight three-factor driven privacy-preserving authentication model specifically for IoT-enabled smart home environments. The authors [51], presented a scheme that automates home appliances in three modes viz: local, web, and app-based automation. These were achieved with a low-cost microcontroller, a web page with support for remote applications. The work [52] presented an improved robots index model used to optimize the robust level of home energy local network (HELN) while considering its numerous household appliances. In [53], a novel proximity service model for smart home automation is proposed. Their work uses wireless networks and native Internet connectivity. The work [54] discussed an asynchronous electrooculography (EOG)based human-machine interface (HMI) for smart home environmental control. The work [55] focused on a machinelearning-based approach to assessing activity quality in smart homes based on automation assessment. The work [56] highlighted an efficient implementation approach using IoT for real-time monitoring of routine activities in homes.

So far, the future of ubiquitous home networks will rely on sensors to aggregate various environmental data variables [57]. This work has similar baseline design attributes with robotic smart homes based on stabilized feedback Episodic memory (SF-EM) [58], stewards robot smart homes [59], humanoid defense smart homes [60], Indoor autonomous robots [61], and Smart home activities IoT [62]. These similarities are highlighted below [63], [64], [65]:

- Process automation through task allocation.

- Lightweight process variable control.

- Layered integrations with NSM.

The identified gaps/limitations are found below:

- Absence of space diversity characterization under multipath fading channels.

- Absence of NSM for hardware analytics.

- Though most systems have complex design topologies.

- Absence of CPS process variable control using the associative-memory based on the reconfigurable memristive neuromorphic scheme.

- The absence of multipath fading channel optimization for memory stabilization is novel.

\section{Summary of Contributions}

The generalized smart home systems are anticipated to provide custom-based services, especially to home users thereby reducing human efforts. Unfortunately, the legacy computational knowledge-driven algorithms for learning and reasoning do not completely orchestrate unpredictable changes in these homes. In this work, the service provisioning demand seeks to handle: i.) remote learning and reasoning process control algorithms, and ii) Edge layer integration of smart endpoints. To fix these issues, the contributions of this work are as follows. 
1) Develop an associative-memory-induced reconfigurable memristive neuromorphic for memory stabilization.

2) Introduce a logical feedback mechanism for behavioral learning from the state machine.

3) Complete a set of space diversity optimization for memory service guarantee from multipath fading errors.

\section{METHODOLOGY}

The physical design topology of the smart automation engine (SAE) is shown in Fig. 1. This depicts the design symmetry of MRASM-ROBOT enabled with machine learning automation and CWDSPT. The implementation of the smart automation/security system is based component layered approach and its subsystems were implemented as a standalone system for ease of reconfiguration. The optimally controlled process variables modeled are temperature states, room lighting, overhead tank, as well as house security.

The design can protect the occupants from life-threatening hazards. This is achieved by getting inputs from sensors placed at different locations throughout the house/deployment environment. Application program interfaces (API) are used for remote communications.

The inputs are then fed into the Arduino MKR1000 Wi-Fi process controller (PCon) through which these different aspects of the house are controlled. Essentially, communication happens in a short-range transmission distance of 100 at $100 \mathrm{~mW}$. This class 1 range uses the RF ISM bands (2.402GHz-2.48GHz).

The objective function (OF) is the infinite process maximization with constraint variables such as link capacity, channel effects, resource availability, and cost function. These are responsible for the smart automation engine (SAE). This further incorporates static real feedback looping for dynamic stability, having an optimal algorithm with low-bit error signaling. These functionalities are implemented to monitor and control devices that drain energy while securely protecting the home facility. As shown in Fig. 1, there are two major parts viz.
- Automation module. This controls the lights, inductive load, and cooling systems (HVACs) in and around the house thereby saving energy. Also, it has the following sub-systems namely - the process controller, level converter, GSM modem, smart green power supply unit, the input interface (sensor subcircuit), control system, and the output Interface such as APIs.

- Associative-memory-induced scheme i.e., memristive reconfigurable state machine attributes.

A brief explanation of the resilient transmitter, RF-wireless communication channel (i.e., transmitter/receiver) for the proposed MRASM-CWDSPT is presented via the component modeling technique. Also, the details proposed CWDSPT, (i.e., DC-biased orthogonal frequency-division multiplexing (DCbiased OFDM) in the IoT controller is equally presented below.

Let's define the following process variables as follows:

$\boldsymbol{A P I _ { g _ { m } }}=$ API gateway

$\boldsymbol{S} \boldsymbol{G} \boldsymbol{g}_{\boldsymbol{V}_{\boldsymbol{\alpha}}}=$ smaoke signal conditioner API communication

$\boldsymbol{W} \boldsymbol{T}_{\boldsymbol{C m}_{\boldsymbol{\beta}}}=$ water signal conditionerAPI communication

$\mathbf{T} \boldsymbol{M}_{\boldsymbol{C} \boldsymbol{m}_{\boldsymbol{\beta}}}=$ Temperature signal controller API communication

$\boldsymbol{L G}_{\boldsymbol{a s}_{\boldsymbol{\mu}}}=$ Light intensity siganl controller API communication

The sink controller functions include Smoke, water, temperature, and light intensity.

\section{A. Memristive Reconfigurable Neuromorphic Circuit Model}

In this section, the component modeling technique was introduced to build the MRASM-Robotic subsystem. This involves process variable synaptic modeling. First, a neuron circuit refers to the schematic baseline unit used to implement a functional neuromorphic system for MRASM-Robotic. It connects more than one synaptic subcircuit interface module depicted in Fig. 1.

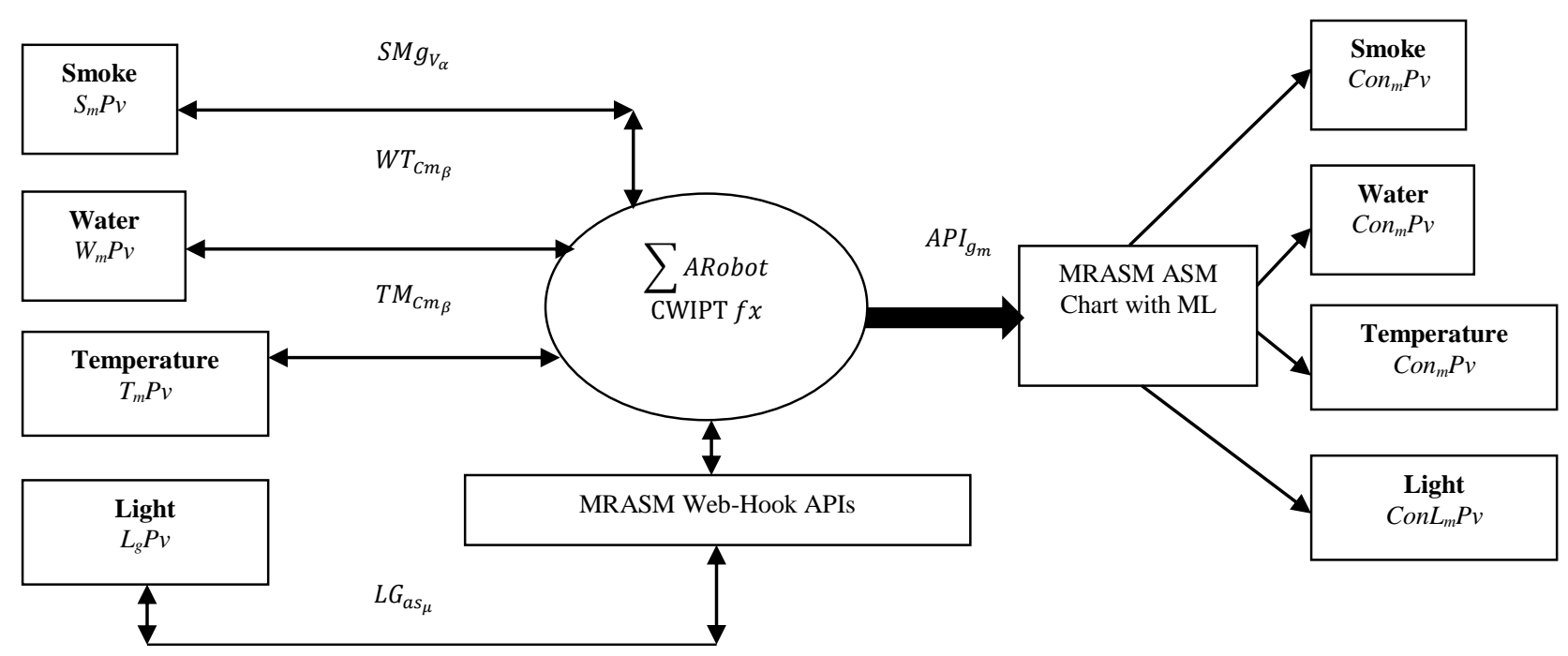

Fig. 1. MRASM-ROBOT Process Model for Memory Stabilization. 
Second, there are three major parts in the neuron circuit: 1) MRASM-Robotic selection circuitry; 2) MRASM-Robotic synaptic circuitry, and 3) MRASM-Robotic neuron circuitry.

In the design implementation, the MRASM-Robotic neuron circuitry has two input voltage levels/signals, MVanalog and MVdigital. The former depicts the analog signal estimated at 1-5 V. The latter refers to the binary digital signal where the voltages of logic 1 and logic 0 are represented as 5 and $0 \mathrm{~V}$ respectively. Voltage thresholds of pMOS transistors and nMOS transistors in MRASM-Robot are fixed at $-0.6 \mathrm{~V}$ and $0.6 \mathrm{~V}$, respectively. $\mathrm{T}_{1}$ and $\mathrm{T}_{2}$ pMOS are used for the selection circuitry.

Now, let's define the computational core of the MRASMRobot which orchestrates both the input and output subsystems in Fig. 1. The process variables (smoke, water, temperature, light signals) modeled in the design include the overhead tank water level sensor module ( $\mathrm{W}_{\mathrm{m}} \mathrm{Pv}$ in Fig. 2a), a Smoke detection module ( $\mathrm{S}_{\mathrm{m}} \mathrm{Pv}$ in Fig. $\left.2 \mathrm{~b}\right)$, temperature control system ( $\mathrm{T}_{\mathrm{m}} \mathrm{Pv}$ in Fig. $2 \mathrm{c}$ ), and motion-controlled lighting ( $\mathrm{Lg}_{\mathrm{g}} \mathrm{Pv}$ in Fig. 2d.).

Using digital comparators shown in Fig. 2a-d, the work estimated appropriate voltages at the output of the comparator. The water resistance was determined experimentally by using a neural network digital ohmmeter (NNDM). A resistance of approximately between $100 \mathrm{k} \Omega$ and $120 \mathrm{k} \Omega$ was obtained using various samples of water. The resistance of $100 \mathrm{k} \Omega$ was taken to be the water resistance using voltage divider models while accounting for tolerance consideration.

A tamper-proof was introduced to monitor open circuit situations or when the sensor goes bad. This is included since if the sensor goes bad without being detected, lives and properties may be lost. The system consists of an additional length of wire connected along with the smoke sensor. This drives the transistor to saturation and diode $\mathrm{D}_{1}$ then conducts. When there is a tamper or if the sensor goes bad, this condition is sensed by the processing unit. From Fig. 2c, ideally, the input of an Op-amp has almost infinite resistance. This then forces the Vcc to be dropped across $R_{30}$ and $R_{31}$. Since the output of LM35 is between $0 \mathrm{~V}$ to $1 \mathrm{~V}\left(10 \mathrm{mV} /{ }^{\circ} \mathrm{C}\right.$ to be precise). To allow power drop across R31, the value was arbitrarily chosen as $1 \mathrm{M} \Omega$. This is well shown in Fig. 2c.

Furthermore, the use of empirical data from machine learning computations is currently investigated in Fig. 1. This is to show the extent of metrics performance between the Memristic control strategy and the traditional models using quantified datasets. Also, The adjusting/changing memristor states are done by changing the resistance value over time during switching of control state variables.

\section{B. Motion Controlled Lighting}

For the LDR section, various experimental trials were carried out. It was noticed that in a dark environment, the LDR will have a resistance of $10 \mathrm{k} \Omega$, thus allowing $2.5 \mathrm{~V}$ to drop across LDR as shown in Fig. 2d. That means that the voltage drop across $\mathrm{R}_{49}=2.5 \mathrm{~V}$. The essence of this sensing arrangement is to create a path across which blockage will obstruct the transmitted rays and the receiving phototransistor, resulting in an open circuit. Anybody entering or leaving the room as a result of crossing this path creates such a situation and will be detected.

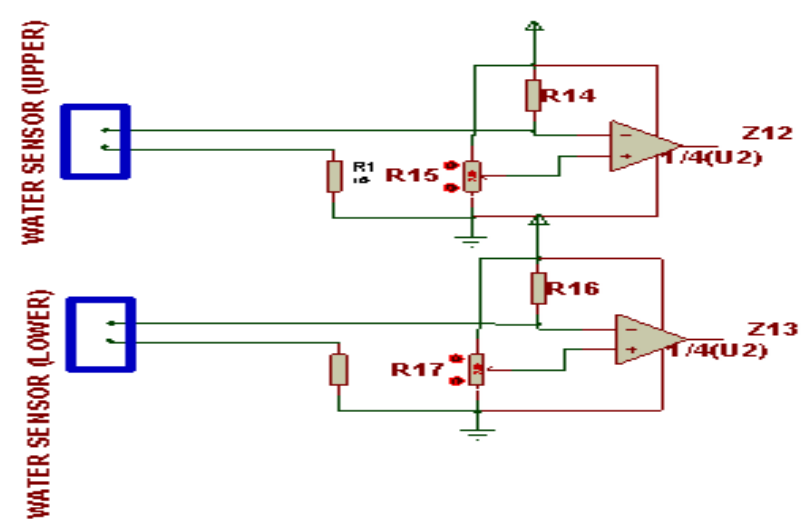

(a)

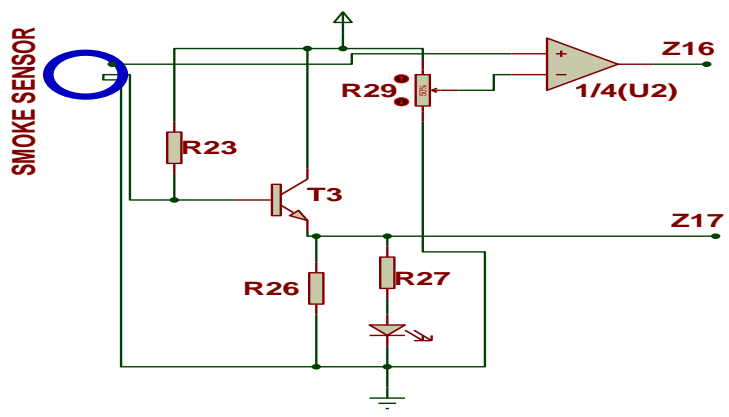

(b)

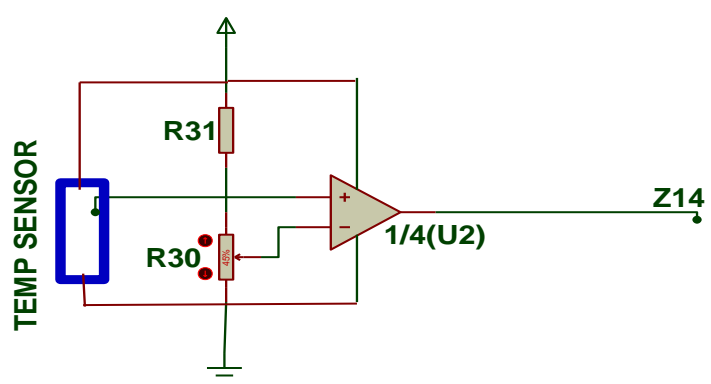

(c)

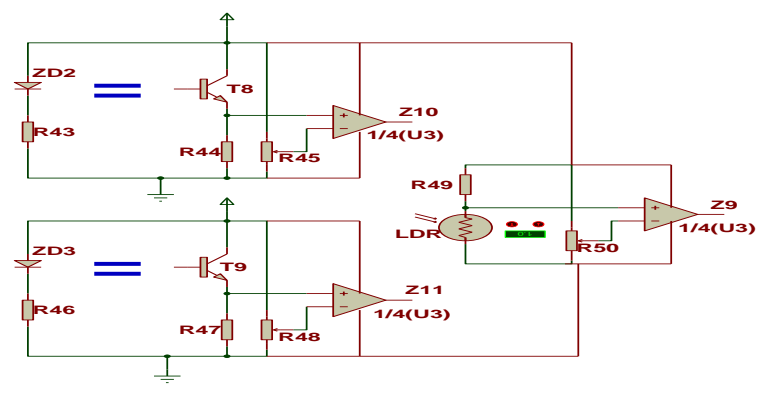

(d)

Fig. 2. (a) MRASM Overhead Tank Sensor, (b). MRASM Smoke Detection Module, (c): MRASM Temperature Sensor, (d). MRASM Motion-controlled Light. 


\section{Pass-worded Locking System}

Push switches are used like keypads for security controls. A switch is used with a resistor as shown in Fig. 3. The value of the resistor selected is $10 \mathrm{k} \Omega$ resistor. When the switch is 'open' the $10 \mathrm{k} \Omega$ resistor connects the microcontroller input pin down to $0 \mathrm{~V}$, which gives an off (logic level 0 ) $0 \mathrm{~V}$ signal to the microcontroller input pin. When the switch is activated, the input pin is connected to the positive battery supply $(\mathrm{V}+)$. This provides an on (logic level 1) signal to the PCon. It is a $4 \times 4$ matrix keypad requiring eight Input/Output ports for interfacing. Rows are connected to peripheral Input/Output (PIO) pins configured as an output. Columns are connected to PIO pins configured as input with interrupts. In this configuration, four pull-up resistors must be added to apply a high level on the corresponding input pins. The corresponding hexadecimal value of the pressed key is sent on four LEDs.

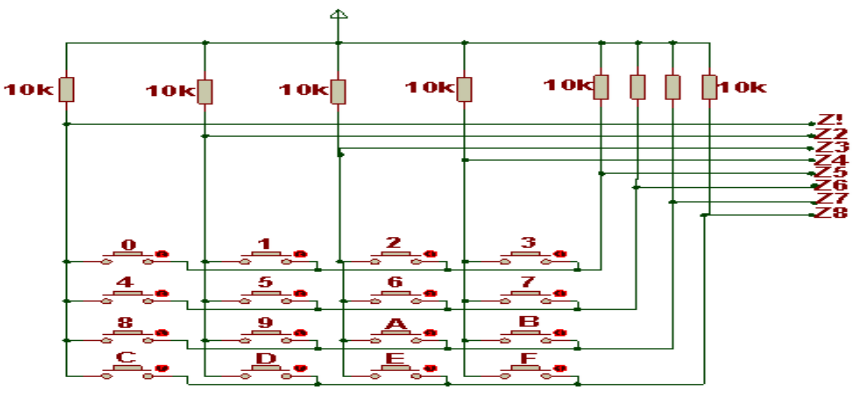

Fig. 3. MRASM-ROBOT Keypad Interface.

\section{SMART Automation EngINE}

In this Section, MRASM-ROBOT was improved with feedback scheduling at the controller level. PIDs algorithm was added for dynamic stability and process controls. The IoT space diversity model for reliable data stream/message delivery is developed. This uses a two-way transceiver communication for memory stabilization. Static real-time scheduling for the automation processes in MRASM-ROBOT was introduced. Also, the design optimization algorithm and implementation emulations, among others were achieved via proofs-of-concept. These components comprise the smart automation engine for MRASM-ROBOT.

\section{A. Design Description}

- Considering the block diagram of the smart automation engine (SAE) in Fig. 1, three layers were identified viz. core, access/automation layer, and aggregation layers. Recall that the process controller is the major component in the core speed redundancy layer, while automation devices in the access layer are interconnected through the ports of the process controller. The MRASM-Robot automation shown in Fig. 4 has the model specifications briefly discussed. There are six process variables in the access layer. These include Water Level Signal ( $\mathrm{P}_{\mathrm{v} 1}$ WLS $)$, Temperature Control Signal ( $\left.\mathrm{P}_{\mathrm{v} 2} \mathrm{TCS}\right)$, Smoke $\operatorname{Signal}\left(\mathrm{P}_{\mathrm{v} 3} \mathrm{SKS}_{-}\right)$, Motion Detection Signal $\left(\mathrm{P}_{\mathrm{v} 4} \mathrm{MDS}\right),=$ Keypad Signal $\left(\mathrm{P}_{\mathrm{v}_{-}} \mathrm{KPS}\right)$. These are all interconnected as in Fig 4. In programming script, $\mathrm{Pv}_{1}, \mathrm{Pv}_{2}, \mathrm{Pv}_{3}, \mathrm{Pv}_{4}$, $\mathrm{Pv}_{5}$ denotes Water Level Signal (WLS), Temperature Control Signal (TCS), Smoke Signal (SKS), Motion
Detection Signal (MDS), and Keypad Signal (KPS) among others. These are all connected to the Process Controller (PCon).

- The process controller uses the optimization algorithm in the aggregation layer to recursively monitor and control the devices without a drop in their stability.

- The MRASM-ROBOT output interface is modeled to report recurrent processes on the system display dashboard or sink. Commands instruction is given to dashboard then execute any predefined task such as initialization, reset, setting the cursor position, controlling the display, etc. The data register stores the data to be displayed on the dashboard. The data is the ASCII value of the character to be displayed on the dashboard.

\section{B. Smart Automation MRASM Chart}

In the MRAM automation system, the derived chart was designed which controls the process variables shown in Fig. 4 using wireless signaling (i.e. CWDSPT). The MRASM chart design is essentially is a finite state machine design scheme used to represent diagrams of process logic regulation in the PCon. The MRASM technique comprises these steps:

1) Create pseudocode for the desired operation of MRASM on the controller.

2) Translate the pseudocode into an MRASM chart.

3) Derive the datapath from the MRASM chart.

4) Create a detailed MRASM chart based on the Datapath.

5) Create the control logic from the MRASM chart.

The outcome of the above steps resulted in Fig. 5 showing the PCon MRASM logic box for the smart automation system. Fig. 5 shows the PCon MRASM logic box for the smart automation system. Fig. 6 shows the design flowchart.

For the model, let the process variables be represented in the MRASM chart State Transition Table presented in Table I with various variables defined below:

$\mathrm{P}_{\mathrm{v} 1}$ WLS $=$ Water Level Signal; $\mathrm{P}_{\mathrm{v} 2 \_} \mathrm{TCS}=$ Temperature Control Signal; $\mathrm{P}_{\mathrm{v} 3}$ SKS $=$ Smoke Signal; $\mathrm{P}_{\mathrm{v} 4}$ MDS $=$ Motion Detection Signal; $\mathrm{P}_{\mathrm{v} 5}$ KPS = Keypad Signal

PSN $=$ Present State Name; PSC $=$ Present State Code $;$ NSN = Next State Name; NSC = Next State Code.

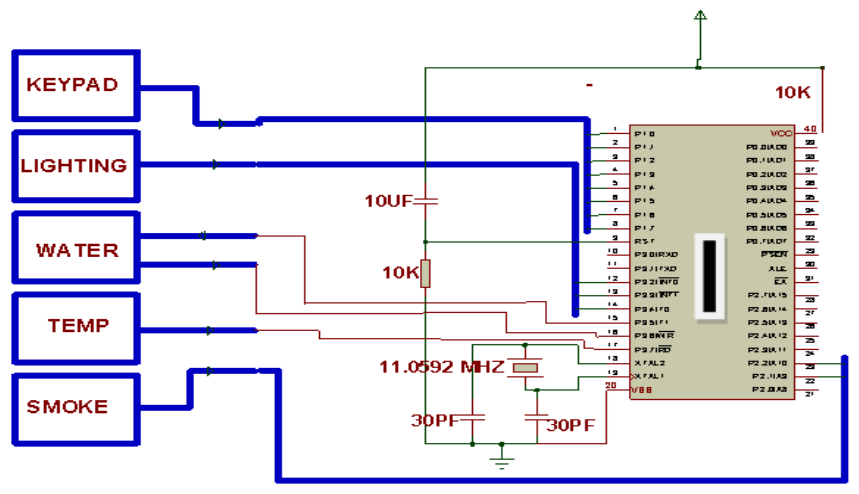

Fig. 4. MRASM-ROBOT Input Interfaces for $\mathrm{Pv}_{1}, \mathrm{Pv}_{2}, \mathrm{Pv}_{3}, \mathrm{Pv}_{4}, \mathrm{Pv}_{5}$, \& $\mathrm{Pvn}$. 
TABLE I. MEMristive ReCONFIGURABle State Machine Transition

\begin{tabular}{|c|c|c|c|c|c|c|c|c|c|c|c|c|c|c|}
\hline \multirow{2}{*}{$\begin{array}{l}\text { LINK } \\
\text { PATH } \\
\text { L1 }\end{array}$} & \multicolumn{5}{|c|}{$\begin{array}{l}\text { Input } \quad \text { Qualifiers } \\
\text { WLS TCS SKS MDS KPS }\end{array}$} & \multirow{2}{*}{$\begin{array}{l}\text { PSN } \\
\text { DCBA } \\
\text { ST0 }\end{array}$} & \multirow{2}{*}{$\begin{array}{l}\text { PSC } \\
0000\end{array}$} & \multirow{2}{*}{$\begin{array}{l}\text { NSN } \\
\text { DCBA } \\
\text { ST0 }\end{array}$} & \multirow{2}{*}{$\begin{array}{l}\text { NSC } \\
0000\end{array}$} & \multicolumn{5}{|c|}{$\begin{array}{l}\text { Output } \\
\text { RL1 RL2 BUZ RL3 RL4 }\end{array}$} \\
\hline & 0 & - & - & - & - & & & & & & & & & \\
\hline L2 & 1 & - & - & - & - & STO & 0000 & ST1 & 0001 & 1 & & & & \\
\hline L3 & 1 & - & - & - & - & ST1 & 0001 & ST1 & 0001 & & & & & \\
\hline L4 & 0 & - & - & - & - & ST1 & 0001 & ST2 & 0011 & 0 & & & & \\
\hline L5 & - & 0 & - & - & - & ST2 & 0011 & ST2 & 0011 & & & & & \\
\hline L6 & - & 1 & - & - & - & ST2 & 0011 & ST3 & 0010 & & 1 & & & \\
\hline L7 & - & 1 & - & - & - & ST3 & 0010 & ST3 & 0010 & & & & & \\
\hline L8 & - & 0 & - & - & - & ST3 & 0010 & ST4 & 0110 & & 0 & & & \\
\hline L9 & - & - & 0 & - & - & ST4 & 0110 & ST4 & 0110 & & & & & \\
\hline L10 & - & - & 1 & - & - & ST4 & 0110 & ST5 & 0111 & & & 1 & & \\
\hline L11 & - & - & 1 & - & - & ST5 & 0111 & ST5 & 0111 & & & & & \\
\hline L12 & - & - & 0 & - & - & ST5 & 0111 & ST6 & 0101 & & & 0 & & \\
\hline L13 & - & - & - & 0 & - & ST6 & 0101 & ST6 & 0101 & & & & & \\
\hline L14 & - & - & - & 1 & - & ST6 & 0101 & ST7 & 0100 & & & & 1 & \\
\hline L15 & - & - & - & 1 & - & ST7 & 0100 & ST7 & 0100 & & & & & \\
\hline L16 & - & - & - & 0 & - & ST7 & 0100 & ST8 & 1100 & & & & 0 & \\
\hline L17 & - & - & - & - & 0 & ST8 & 1100 & ST8 & 1100 & & & & & \\
\hline L18 & - & - & - & - & 1 & ST8 & 1100 & ST9 & 1101 & & & & & 1 \\
\hline L19 & - & - & - & - & 1 & ST9 & 1101 & ST9 & 1101 & & & & & \\
\hline L20 & - & - & - & - & 0 & ST9 & 1101 & ST0 & 0000 & & & & & 0 \\
\hline
\end{tabular}

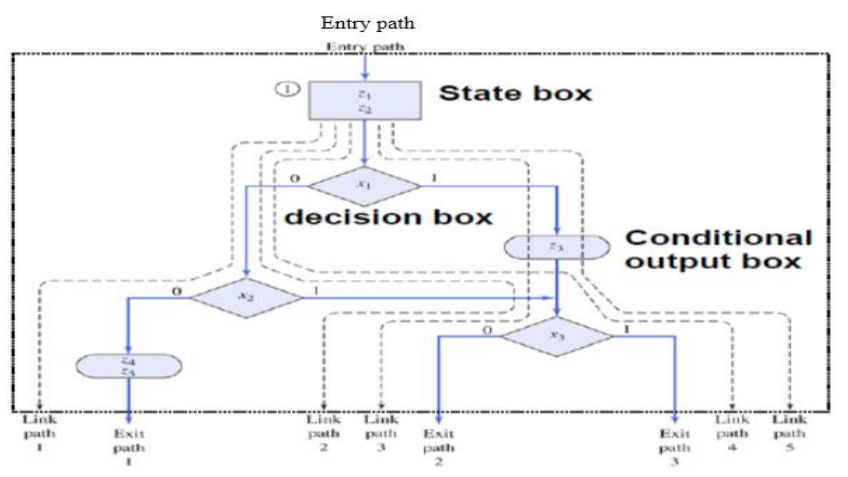

Fig. 5. MRASM Process Controller Block.

\section{MRASM Design Description}

As depicted in Table I, the design chart for MRASM smart automation security control was further characterized using schematics capture C++ scripting. Test case values are derived from optimal system response conditions. The input interface to the PCon is made up of the input qualifiers. The qualifiers are the comparator output from the different sensor modules at the access layer. As shown in Fig. 6, these include the water level signal $\left(\mathrm{P}_{\mathrm{v}_{1}} \mathrm{WLS}\right)$, smoke signal $\left(\mathrm{P}_{\mathrm{v}_{3}} \mathrm{SKS}\right)$, temperature control signal ( $\left.\mathrm{P}_{\mathrm{v}_{2}} \mathrm{TCS}\right)$, the motion detection signal $\left(\mathrm{P}_{\mathrm{v} 4} \mathrm{MDS}\right)$, and the keypad signal ( $\left.\mathrm{P}_{\mathrm{v}_{5}} \mathrm{KPS}\right)$. Whenever any of the qualifiers changes its state, either from logic 1 to logic 0 or from logic 0 to $\operatorname{logic} 1$; there is an event at the output interface. For instance, in link path L1 to L4, when the input qualifier, water level signal (WLS) changes its state from logic 0 to logic 1 (L1-L2), Relay1 (the relay that turns on the pumping machine) is energized and the water pump turns on. As long as it stays on in 1 , the water pump will keep on pumping water. But once it changes its state from 1 to 0 (L3-L4), Relay1 is deenergized and the water pump turns off.

The link path L5 to L8 depicts what happens in the temperature channel and how the microcontroller reacts to it. When the temperature exceeds the preset value or goes below the preset value, the signal from the temperature module changes from logic 0 to logic1 (L5-L6), Relay 2 is energized and the air conditioner turns on. On the other hand, when the signal goes from 1 to 0 (L7-L8), the relay is de-energized and the air conditioner turns off. The L9-L12 depicts what happens in the smoke channel how the microcontroller reacts to it and what happens at the output interface. When there is a smoke occurrence, the signal from the smoke channel (one of the input qualifiers), changes from logic 0 to 1 L9-L10, the Buzzer is energized and turned on. For as long as the signal remains in logic 1 , the buzzer will be sounding, but when a signal goes from logic 1 to 0 (L11-L12), the buzzer is de-energized and therefore stops sounding.

The link path L13-L16 depicts what happens in the motioncontrolled light module and how the microcontroller reacts to it. When motion is detected and there is insufficient light, the signal from this module changes from logic 0 to 1, L13-L14, the relay known as RL3 is energized and the light turns on. As long as the person is in the room, the light will be on. But once the person leaves, that is, when the person is no longer sensed, the signal changes from logic 1 to 0, L15-L16 RL3 is deenergized and the light turns off. 


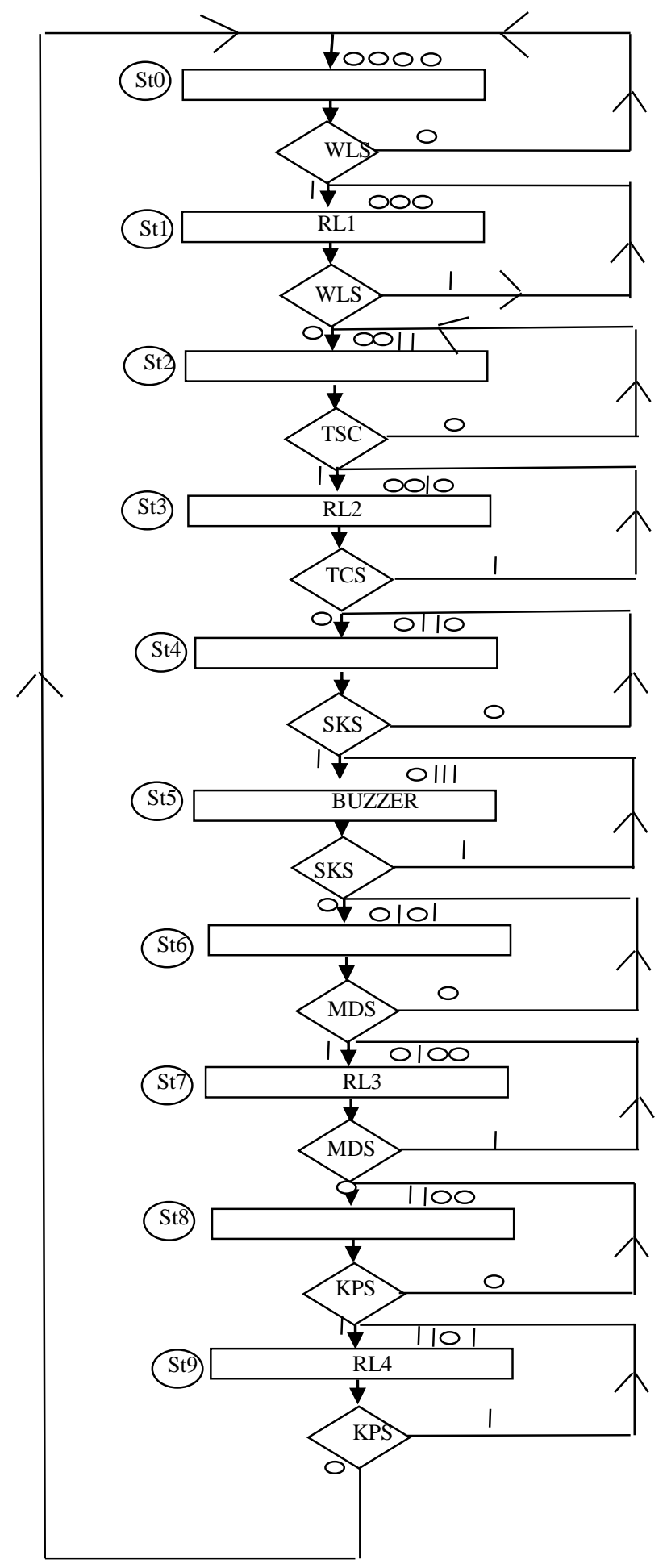

Fig. 6. MRASM Design Chart for Smart Automation and Security Design System.

The link path L17-L20 depicts what happens when the keypad is pressed. When pressed and the input code is correct, L17-L18, the signal from here changes from logic 0 to 1 , the relay known as RL4 is energized and the door opens. Once the door is shut, the signal changes from logic 1 to 0, L19-L20. With the MRASM Chart and the corresponding, STT table, the system, home automation, and security system were developed in the prototype design. The keypad signal $\left(\mathrm{P}_{\mathrm{v} 5} \mathrm{KPS}\right)$ goes into the PCon through port 3 pins 1 to 8 of the controller chip. The motion detection signal ( $\left.\mathrm{P}_{\mathrm{v} 4} \mathrm{MDS}\right)$ goes into the PCon through port 3 pins $12,13,14$. The water level signal $\left(\mathrm{P}_{\mathrm{v} 1}\right.$ WLS $)$ goes into the PCon through port 3 pins 15 and 16. The temperature control signal ( $\mathrm{P}_{\mathrm{v} 2}$ TCS $)$,) goes into the PCon through pins 17. While the smoke signal $\left(\mathrm{P}_{\mathrm{v} 3}\right.$ SKS $)$ goes in through pins 22 and 23. Fig. 7 shows the neuromorphic/schematic implementation described above.

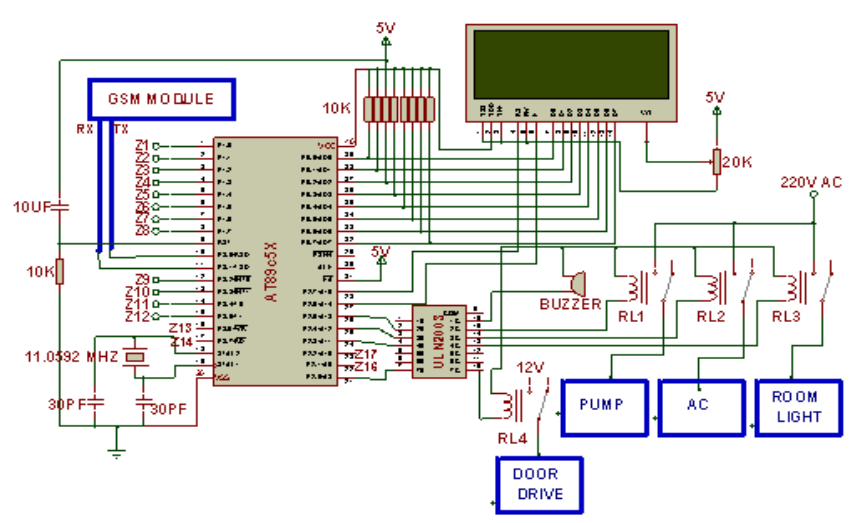

Fig. 7. MRASM-ROBOT Unified Schematics.

\section{FEEDBACK SCHEDULING AlgORITHM}

\section{A. Dynamic Stability Control}

Dynamic stability was introduced using digital real-time scheduler architecture in Fig. 8. This is implemented in Algorithm I, and II. The scheduler links the internal feedback control loop structures synchronously. Also, it sets the error margin for the scheduler, observes the error deviation state, and dynamically adjusts the infinite process variables for stable optimization. Algorithm II shows the temperature optimization algorithm. The AC, lights, pumps, and other processes are continuously monitored for efficient power management. At each instance, a control API communication is triggered for remote transmission.

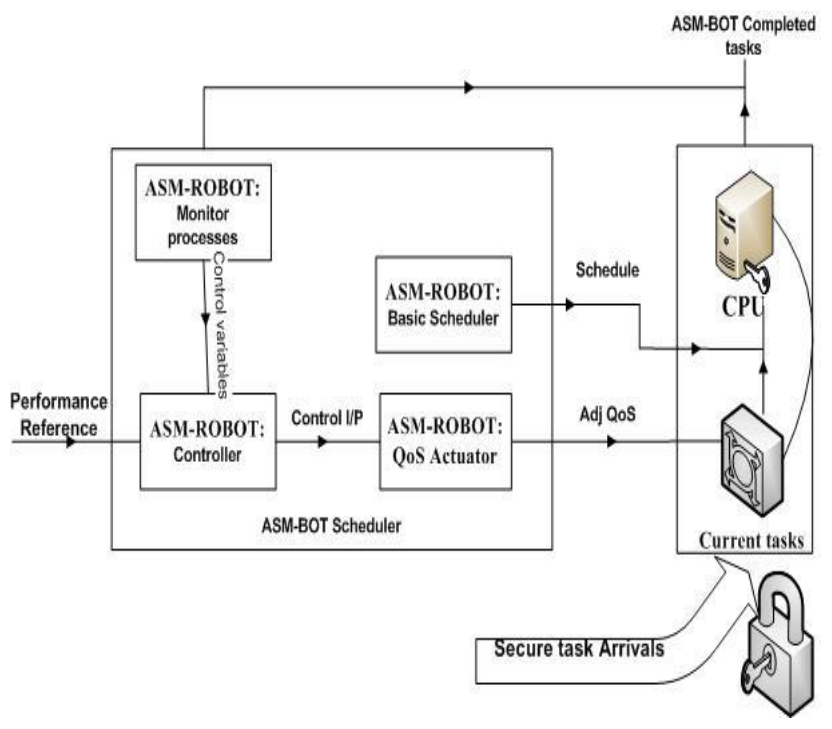

Fig. 8. MRASM-BOT Scheduler Architecture. 
Internally, the deadline, estimated execution time, actual execution time, and baseline tasks are processed once the performance reference is set via initialization procedures. The MRASM-BOT captures the infinite process variables in the system and feeds samples to the controller. The controller makes comparisons between the performance reference and the controlled variables to ascertain immediate errors, and compute any error differential. The QoS actuator smartly adjusts the computed utilization at each sample scenario. The algorithm I demonstrate the optimization controls.

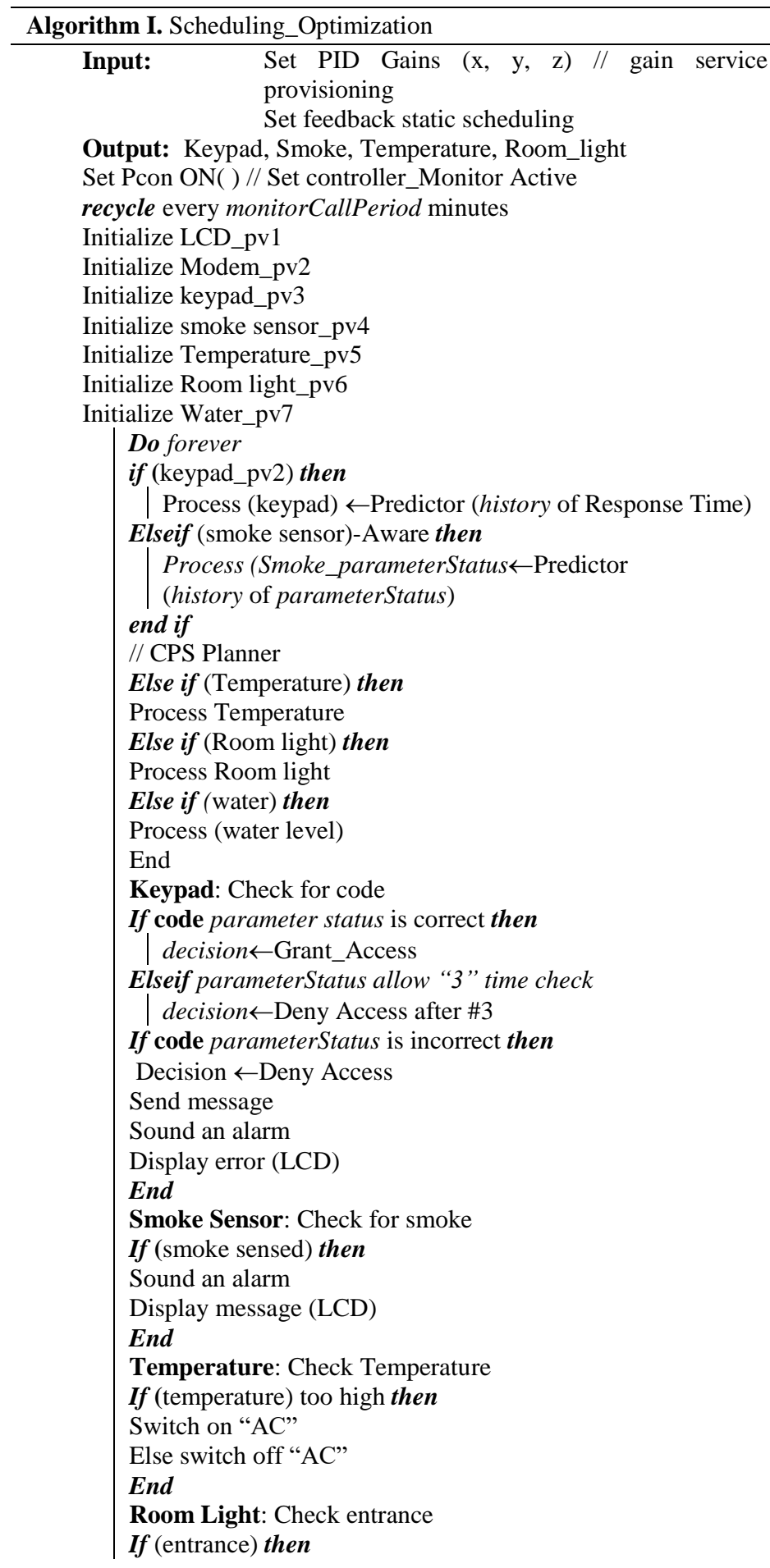

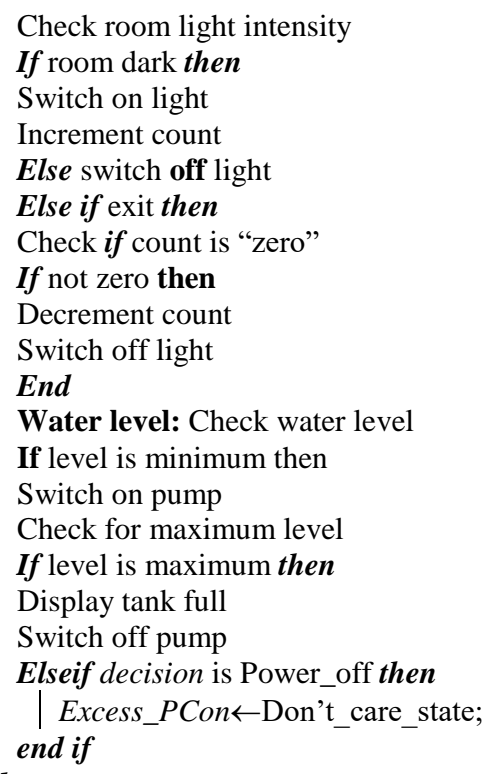

In the neuromorphic automation model, once the message is sent, the message is received by SMSC (SMS controller). This then reaches an appropriate device/interface. Recall that the process controller (PCon) interface for SMS in Algorithm I provides a path for transmitting control signals in full-duplex mode.

A simple algorithm for IoT-SMS communication in the smart automation model is given in Algorithm II.

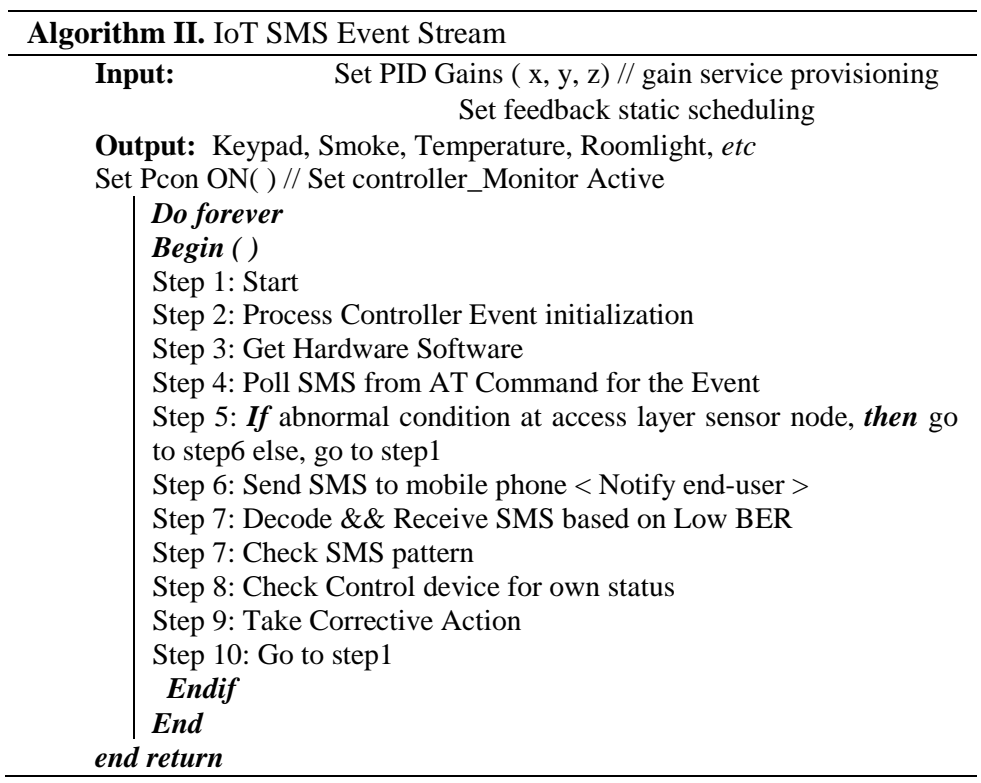

\section{B. Reconfigurable Associative-Memory}

Recall from Table I, the memristive neuromorphic scenario is derived completely for all the machine states. The neuron circuitries with associative-memory-based reconfigurable neuromorphic (AMBRN) structure are implemented. In this case, learning and forgetting with associative memory dynamically reconfigures the circuit schematics. For this MRASM design, the design structure and its use-case are shown in Fig. 9. 


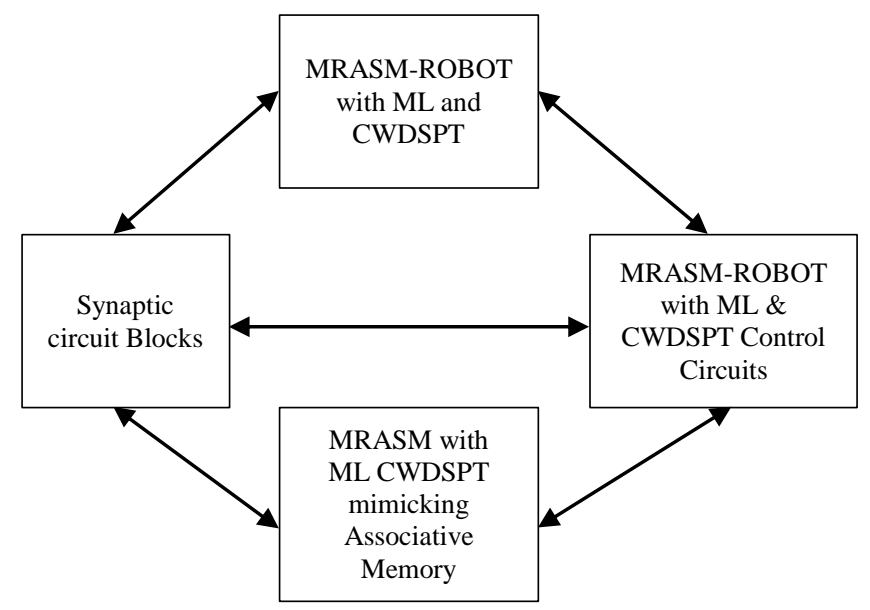

Fig. 9. Neuromorphic System Structure.

In Fig. 9, the AMBRN system has the following entities: 1) Machine learning subsystem block for speeding up process controls; 2) associative memory mimicking subsystem; 3) synaptic circuit block, 4) control circuits. The technique of mimicking the associative learning and the remembrance processes between conditioned and unconditioned process variable signals achieves the activation function. The remembrance process allows the control circuits to activate or deactivate links to the synaptic circuits. These synaptic circuits implement ML optimization in the robot, though this is currently under investigation. It realizes the associative memory network for dynamic responses.

\section{SPACE DiVERSITY CONTROL MODEL}

\section{A. IoT RF Modulation Construction}

In this research, an investigation into the design properties of IoT RF transceiver was carried out in [66] leading to the model for computing error performance by measurements in Fig. 10. The model accounts for:

- Receiver sensitivity with different error measures (BER, Error SNR).

- Modulator frequency and phase error.

- Timing error deviation of the IoT RF transceiver.

Considering Fig. 10, the MRASM digital modulation process encodes data stream information from the sensed sources and makes it suitable for transmission. The modulation technique transports available data stream message/signal via radio channel. Best transmission quality on an optimal radio spectrum is leveraged.

Let's now, consider the SMS message from the robot as:

$\psi(t)=\beta \cos (w t+\theta) \rightarrow$ Channel

Where $\psi(\mathrm{t})=$ data streams, $\beta \cos (w \mathrm{t}+\theta)$ is the modulation. It is key to note that modulation is achieved via amplitude $(A=\beta)$ variation, phase $\theta$, high-frequency carrier (wt) per the amplitude of the data streams signal. Also, Complexity Minimum Shift Keying (CMSK) is used for IoT $\mathrm{RF}$ optimization. The reason is that it offers a uniform envelope, optimal spectral efficiency, excellent bit error rate.

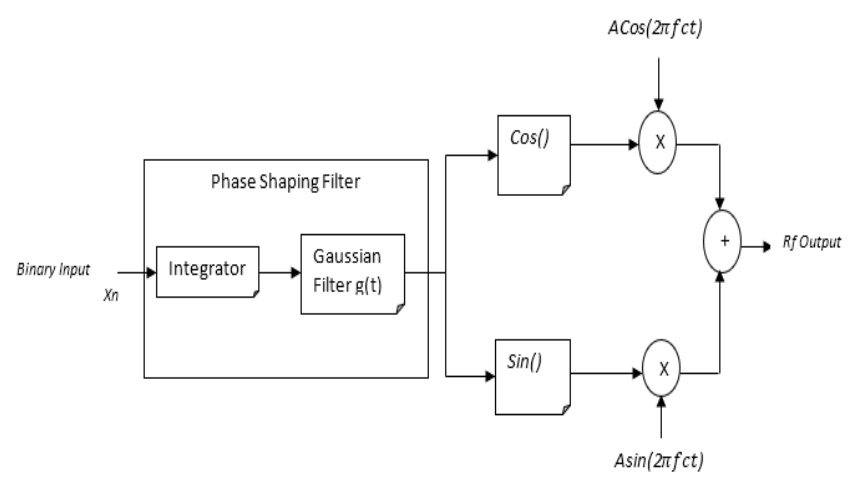

Fig. 10. MRASM IoT Modulation.

In context, the mathematical model for the modulated function $X(t)$ is shown in (2).

$x(t)=\operatorname{Cos}\left(2 \pi f_{c}+\phi(t)\right)$

$\phi(t)=2 \pi h \int_{-\infty}^{-\infty} \sum_{k=-\infty}^{-\infty} a_{s} s(\tau-k T) d \tau$

\section{B. IoT GSMK Modulator}

In the RF diversity design for MRASM-ROBOT in Fig. 10, complex IoT GSMK modulator/demodulator was used which has a Gaussian frequency shaping filter and key parameters from orthogonal space-time block code (OSTBC) transmission [66], sideband modulation [67], continuous phase modulation [68], GFSK Receiver [69], GMSK Pulse [70]. The scheme offers a continuous phase modulation (CPM) signal and has a modulation index $h=1 / 2$. This means that the continuous phase shift function $\theta(\mathrm{t})$ will have the complex baseband structure shown in (4).

$\tau_{\text {transmit }}(t)=\beta \exp \sum_{n}^{1} x_{n} \phi(t-n T)+\phi_{0}$

Where $T$ is bit period, $\beta$ is amplitude, $X_{n}= \pm$ is the structural binary symbols, $\phi_{0}$ is the random initial phase and $\phi(\mathrm{t})$ is the phase shift function.

The unmodulated continuous-wave technique employed is robust and is not affected by signal fading and interference. Its spectral efficiency is optimal with slower/smoother phase changes. From Fig. 9, the IoT modulation signal is obtained through modulation and infusion of two quadrature carriers having frequency $\boldsymbol{F}_{\boldsymbol{c}}$. Phase changes are smoothed with a filter whose Gaussian impulse response is given by (5):

$g(t)=\frac{1}{2 T} Q 2\left(\pi B \frac{t-\frac{T}{2}}{\sqrt{\ln 2}}\right)-Q\left(2 \pi B \frac{t+\frac{T}{2}}{\sqrt{\ln 2}}\right)$

Where $Q(t)$ is the Q-function given by (6):

$Q(t)=\int_{t}^{\infty} \frac{1}{2 \pi} \exp \left(-\frac{r^{2}}{2}\right) d r$

and the phase shift function $Q(t)$ in (1) is given by (7):

$\phi(t)=\int_{-\infty}^{t} g(t) d t$

In all, the IoT bandwidth and interference resistance parameters are controlled within space diversity by combination. The IoT modulation interface is shown in Fig. 11a. The source Bernoulli input is fed into the IoT RF 
transmitter comprising convolutional encoding, data framing, interleaving, data burst, and cyclic redundancy checks (CRC). Also, its differential encoder with GMSK modulator is optimized for resilient transmission of process variables. The $\mathrm{RF}$ channel is constructed using additional white Gaussian noise (AWGN) and the Multipath Rayleigh fading. These were introduced for BER testing.

Similarly, the receiver the demodulation is derived from a decoder that has an isolated matched-filter, cyclic redundancy check, decoder, GMSK demodulation, differential-decoder, and reshape-optimizer. The receiver interface terminates with a BER/Error estimator sink.

In this work, BER is processed additive mapping, i.e., including source generated data with the demodulator output. Using GMSK modulation and demodulation processes in Fig. 11a leads to a stable but sensitive system. Form Fig. 10, the base BER is 0.03846 (i.e. less than 1). Hence, the system space diversity achieves absolute reliability as a transceiver unit.

In the MRASM-ROBOT, complex multiple-input multipleoutput (MIMO) deployment especially in Rayleigh fading, can be determined.

Let's consider a complex IoT MIMO scenario with $\mathrm{N}_{\mathrm{s}}$. Transmit antenna and $\mathrm{N}_{\mathrm{d}}$ receive antennas, where $\mathrm{N}_{\mathrm{s}} \mathrm{N}_{\mathrm{d}} \in$ $Z^{+}, \mathrm{N}_{\mathrm{d}}>N_{s}$, and $\mathrm{N}_{\mathrm{d}}$ and $N_{s}$ use huge values. The OSTB transmission for complex constellations in $\mathrm{N}_{\mathrm{d}} * N_{s}$ MIMO system is derived from [66]. The MRASM-ROBOT channel matrix $\mathrm{H} \in \mathrm{CNd}^{*} \mathrm{Ns}$ can be computed from (8).

$\mathrm{Y}=\mathrm{HC}+\mathrm{W}$

where $C$ is the is code matrix, transmitted from transmitter and

$W$ is additive white Gaussian noise (AWGN) noise matrix.

By transforming (8) such that IoT OSTBC receiver combiner interface is shown in Fig. 11(b). The orthogonal code matrices $C$, decoder design are derived from [66]. The results for OSTBC are discussed later in Section VII.

Again, let's consider a single-IoT RF diversity design for which the signal received in (8) is the sum of the expected data stream signal and noise $(W=n)$ given by:

$X=h u(t)+n$

Where $C=u(t)$ denotes IoT transmitted power signal. $H=$ $h$ denotes channel matrix with signal power. $\mathrm{n}$ the noise. The signal power sent out over a period, $T s$, at nth element is given by (10).

$P=\frac{1}{T_{S}} \int_{0}^{T_{S}}\left|h_{n}(t)\right|^{2}|u(t)|^{2} d t=\left|h_{n}(t)\right|^{2} \frac{1}{T_{s}} \int_{0}^{T_{S}}|u(t)|^{2} d t=$ $\left|h_{n}\right|^{2}$,

Assuming slow fading due to small distance $<50 \mathrm{~m}$, the term $\left|h_{n}(t)\right|^{2}$ will be kept constant for a period with an integral unit power $\mathrm{E}$.
By letting $E\left\{\left|n_{n}(t)\right|^{2}\right\}=\sigma^{2}$, the instantaneous SNR at the $n$-th element $\left(\gamma_{n}\right)$ is given by (11).

$\gamma_{n}=\frac{\left|h_{n}\right|^{2}}{\sigma^{2}}$

The instantaneous SNR under channel matrix hn is estimated such that noise power is obtained over a relatively short period $\mathrm{T}$.

Given that Rayleigh fading is still possible over shortrange, hence, $h_{n}=\left[h_{n}\right] e^{j \angle h_{n}}$, where $\angle h_{n}$ is constant in $(0,2 \pi) .\left[h_{n}\right]$ still has Rayleigh pdf, such that $\left|h_{n}\right|^{2}$ and $\left(\gamma_{n}\right)$ has an exponential pdf given by (12).

$\left[h_{n}\right] \sim \frac{2\left[h_{n}\right]}{P_{0}} e^{-\left|h_{n}\right|^{2} / P_{0}}$

$\gamma_{n} \sim \frac{1}{\Gamma} e^{-\gamma_{n} / \Gamma}$

$\Gamma=E\left\{\gamma_{n}\right\}=\frac{E\left\{\left|h_{n}\right|^{2}\right\}}{\sigma^{2}}=\frac{P_{0}}{\sigma^{2}}$

Therefore, the instantaneous SNR at individual RF elements is exponentially distributed and $\boldsymbol{r}$ denotes the average SNR at each IoT element. This is also the SNR of a single element IoT RF antenna, i.e., the SNR with zero arrays. Hence, $r$ is now the baseline for IoT RF SNR enhancement Fig. 11(b).

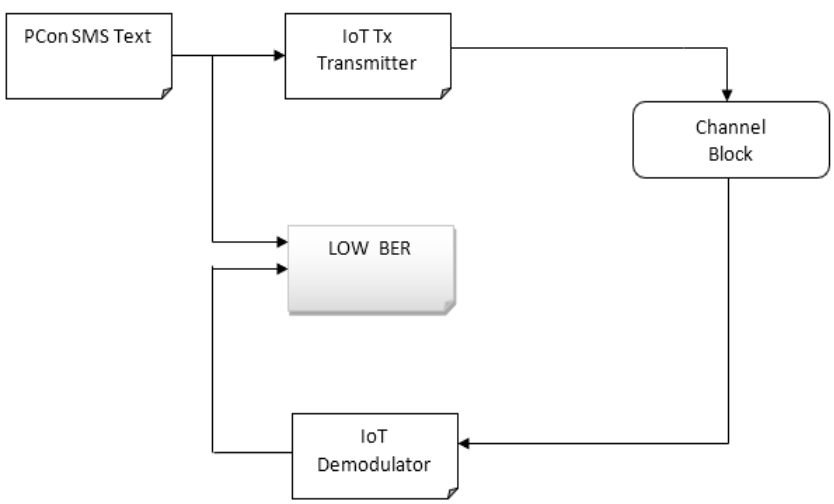

(a)

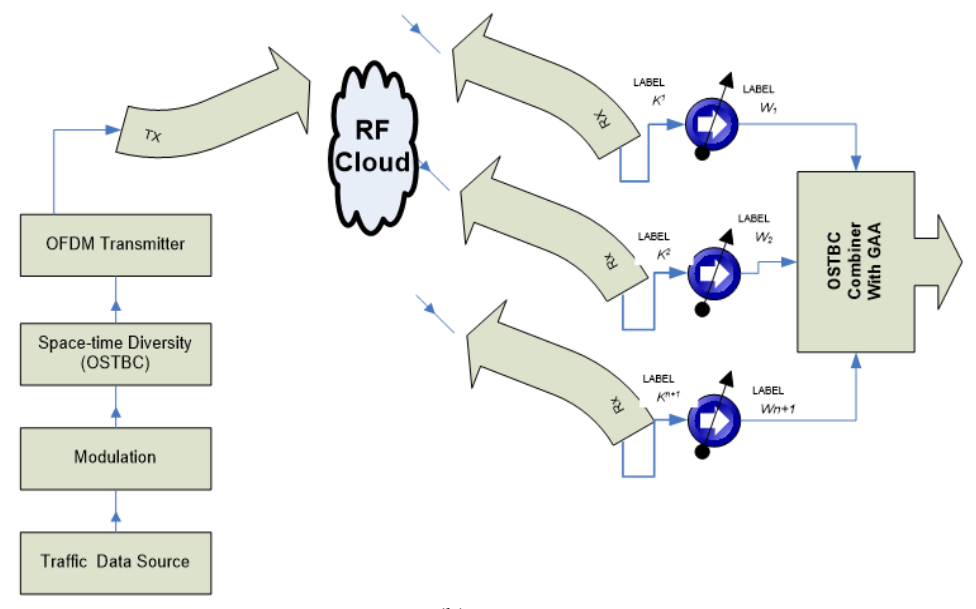

(b)

Fig. 11. (a) IoT Modulation Interface Model., (b). IoT OSTBC Receiver Combiner. 


\section{VII.EXPERIMENTAL RESULTS}

\section{A. MRASM-ROBOT Transmitter-Receiver Analysis}

In this section, the experimental results to verify the proposed MRASM for the neuron control variables using space diversity theorems are presented. A brief discussion on the performance of the IoT CWDSPT scheme is highlighted. As a first step, $\mathrm{C}++$ scripts was used to implement the control logic while MATLAB tool generated the plots. The implementation of the final version of the MRASM-ROBOT is currently undergoing packaging as a commercial off-the-shelf derivative.

For the IoT module, the receiver circuit is mapped at the carrier frequency $\mathrm{FC}=2.4 \mathrm{GHz}$. These schematics depict both embedded transmitter and receiver circuitry of the IoT module respectively. In Fig. 12a, the MRASM-ROBOT depicts the smart home automation and security system in an off-mode scenario. In Fig. 12b, the MRASM-ROBOT depicts the smart home automation and security system in the ON mode scenario.

\section{B. OSTBC Optimization Response}

Recall in Section VI, the IoT GSMK Modulator was introduced. For process variables, the CWDSPT are affected by Multipath fading effects especially IoT RF interface that works with channel interference and AWGN. This paper introduced an enhanced layer 2 protocol called OSTBC. It is based on orthogonal frequency division multiplexing (OFDM) to suppress and enhance traffic frame delivery in the MRASM design. The OSTBC strategy in the receiver diversity block regulates efficient throughput by suppressing channel effects and recovering the transmitted signals.

Fig. 12 shows the error low pass filtering effect for noise reduction at receiving node while Fig. 13 shows the declining probability bit error rate (BER) under channel noise effect. These plots show the optimal capacity of the IoT CWDSPT scheme. This is because signal transmission and reception from other modules are achieved seamlessly.

The implication is that the MRASM-Robot supports process variable manipulation while reducing both the RF interference level (MRCSV) and (AWGN) for optimal performance.

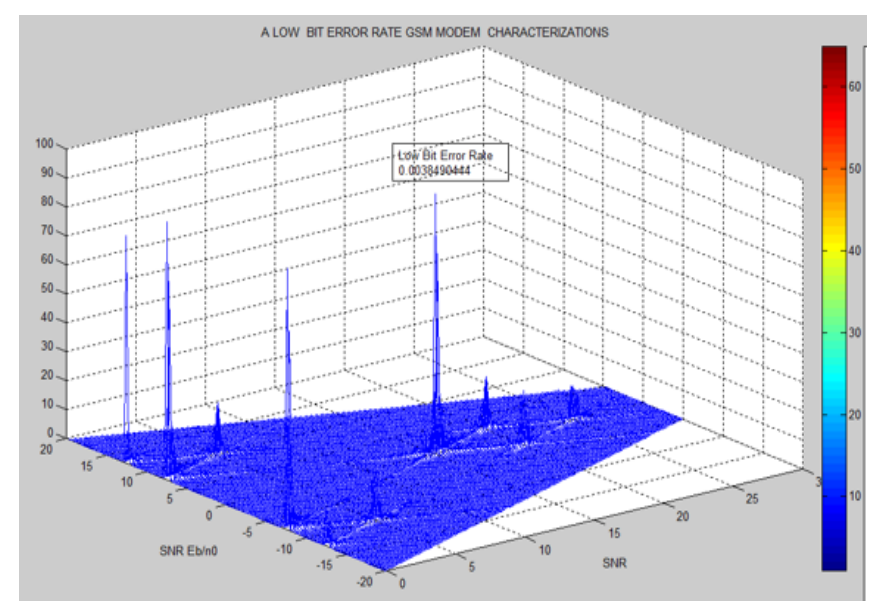

Fig. 12. MRASM-ROBOT Error Deviation with IoT Nodes.
Based on data generated, the relationship model using the modulation technique for BER is depicted in Table II. BER graph was obtained considering the AWGN channel and multipath fading. The GSMK modulation technique in the AWGN channel has good performance as shown in Fig. 12. When the robot was placed in the Multipath Rayleigh channel. an increasing value of Doppler shift $(\mathrm{Hz})$ is shown to be acceptable. The implication is that the system will perform poorly as the coverage distance of the robot RF terminal is increased. Moreover, the system performs average well since the BER is quite low for such short-range communication distance. The results for BER vs. SNR are summarized in Table III.

TABLE II. SimUlation SPECIFICATION [71]

\begin{tabular}{|l|l|}
\hline Simulation Parameters & Specifications \\
\hline Scenario & Macro-Cell, 7-Nodes B's (21 Sector) \\
\hline Bs-2-Bs Distance & $2800 \mathrm{~m}$ (Large), 100m \& 10m \\
\hline Cell Radius & $933 \mathrm{~m}$ \\
\hline Propagation Model & OSTBC Space diversity \\
\hline Channel Profile & Multipath fading +AWGN \\
\hline Modulation & OFDM bank \\
\hline Channel bandwidth & $3.5 \mathrm{MHz}$ \\
\hline OFDM Symbols per burst & 2 \\
\hline Cyclic Prefix Factor $(\mathrm{G})$ & $1 / 8$ \\
\hline Receiver Type & OFDM Receiver \\
\hline Max.Doppler Shift (Hz) & 0.5 \\
\hline Gain vector (db) & {$[0-5-10]$} \\
\hline Initial Seed & 61 \\
\hline Channel SNR & 20 \\
\hline Number of Simulations & 100 \\
\hline Traffic Model & Infinite Buffer \\
\hline
\end{tabular}

. . . Bit Error Rate(BER)

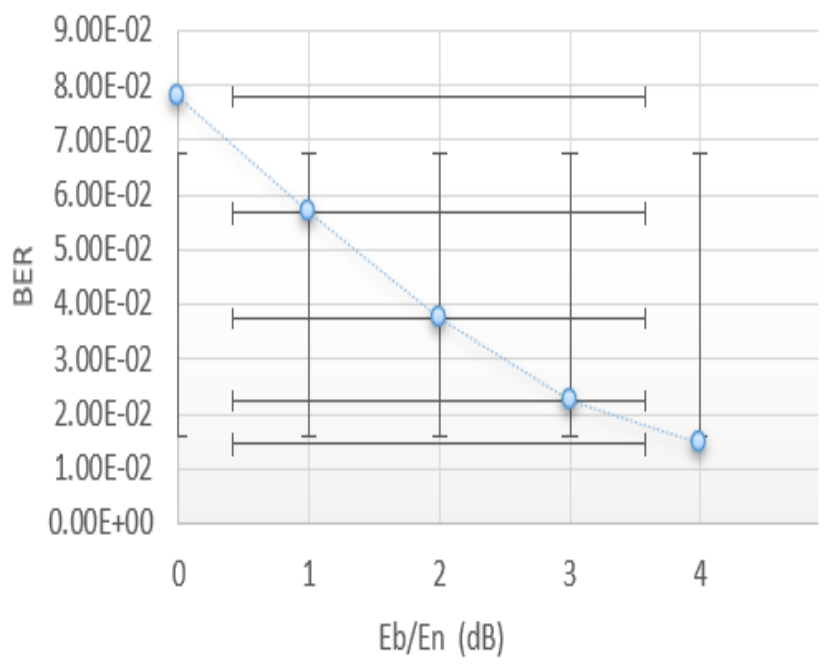

Fig. 13. Response Plot of MRASM-ROBOT under Channel Effects. 
TABLE III. ERROR VALIDATION RESULTS

\begin{tabular}{|l|l|l|}
\hline $\mathrm{SNR} / \mathrm{E}_{\mathrm{b}} / \mathrm{E}_{0}$ & Number of Errors & Bit Error Rate (BER) \\
\hline 0 & 15615 & $7.81 \mathrm{E}-02$ \\
\hline 1 & 11334 & $5.67 \mathrm{E}-02$ \\
\hline 2 & 7520 & $3.76 \mathrm{E}-02$ \\
\hline 3 & 4481 & $2.24 \mathrm{E}-02$ \\
\hline 4 & 2489 & $1.44 \mathrm{E}-02$ \\
\hline
\end{tabular}

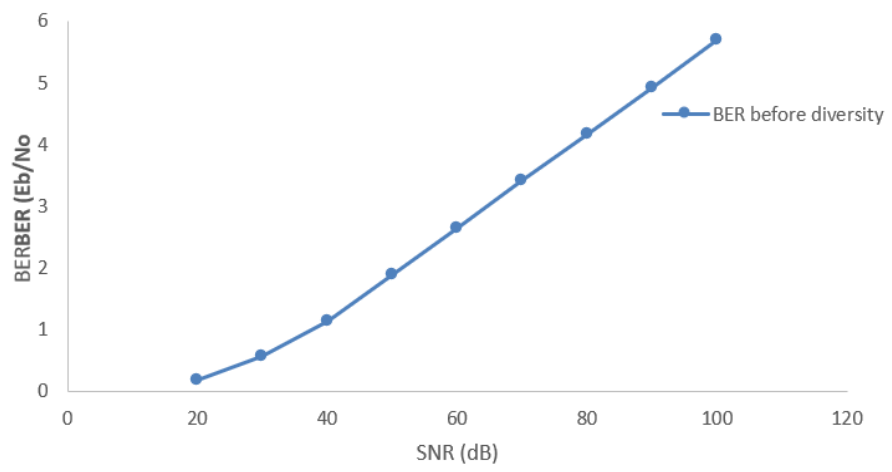

Fig. 14. MRASM-ROBOT BER before Diversity with IoT Nodes.

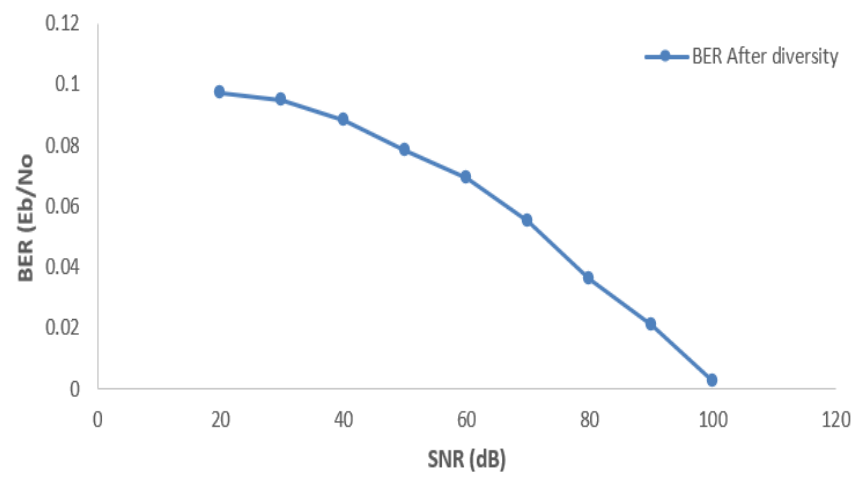

Fig. 15. MRASM-ROBOT BER after Diversity with IoT Nodes.

MRASM-ROBOT MIMO module is investigated for spatial diversity analysis since the robot communicates from a source to a sink always. The additive noise is varied with SNR. Space diversity analysis was carried out in Fig. 14 depicting ER before diversity and Fig. 1 denoting BER after diversity. Fig. 15 increases the reliability of the MRASM-ROBOT MIMO module. A second MRASM-ROBOT MIMO module (i.e., the IoT diversity antenna) below the first (i.e., the primary antenna) at each location of the MRASM-ROBOT having MIMO module will increase reliable connectivity.

Fig. 16, 17, 18, 19, and 20 shows that with the space diversity, the model will scale gracefully while offering better performance than the multipath fading schemes.

Under concurrent wireless data streams and power transfer, various space diversity simulation runs were executed and obtained polar graphs with different scenarios of CWDSPT (optimized and un-optimized). The analysis considered multipath channel fading effects in IoT RF interfaces. In the receiver diversity, with the channel interruptions, the plot no
OSTBC combiner is shown in Fig. 18 and 20. This leads to multipath fading channel issues and there is no memory stabilization since the OSTBC combiner block is absent. Fig. 19 and 21 depict highly optimized memory stabilization for the MRASM-ROBOT process variables. The optimization scenarios reflect the parametric sensitivities highlighted in Table III.

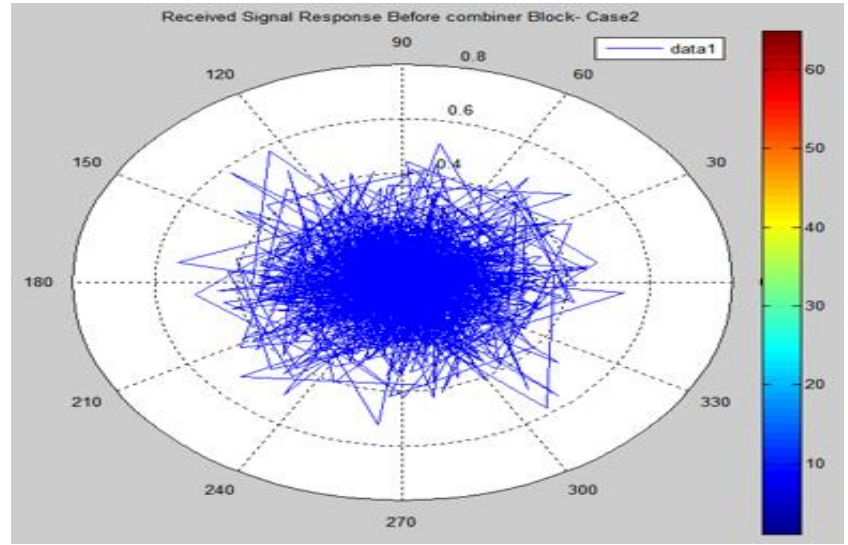

Fig. 16. Polar Plot of Received Signal without OSTBC Combiner Block (Case-2)

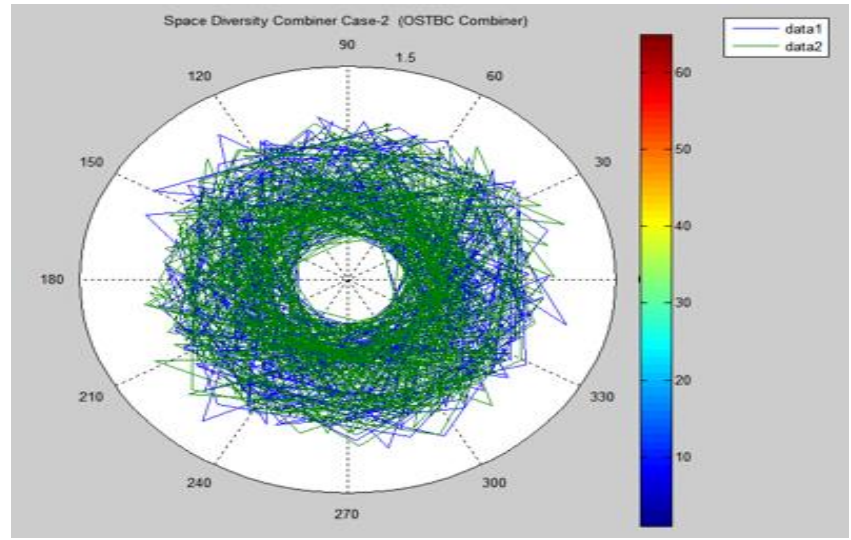

Fig. 17. Polar Plot of Received Signal with OSTBC Combiner Block, Case-2.

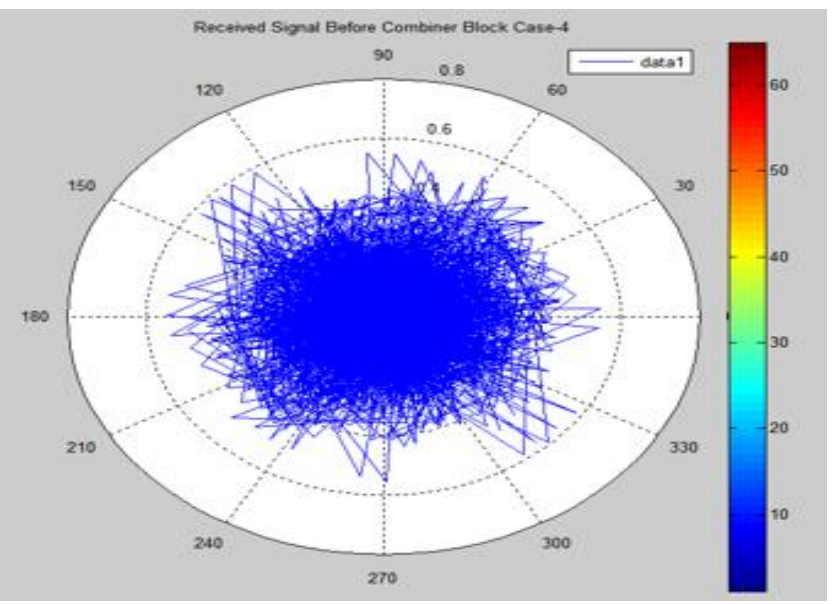

Fig. 18. Received Signal without Combiner Block, (Space Diversity Combiner Case -4). 


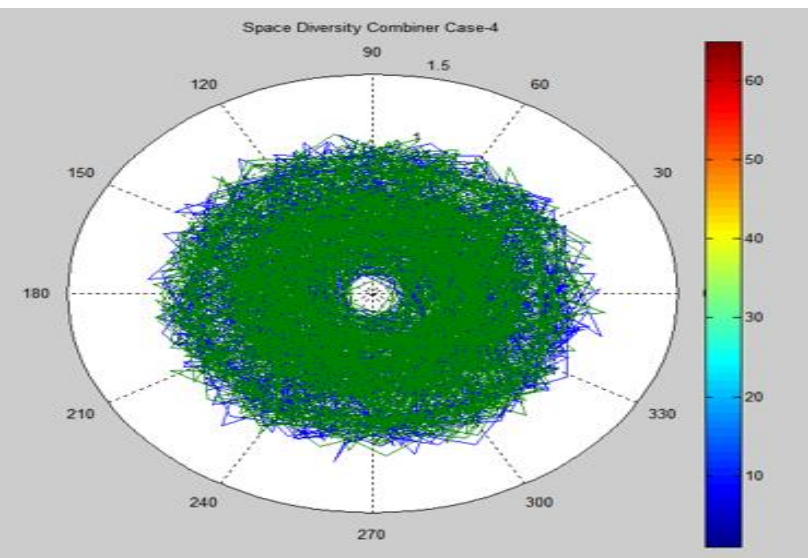

Fig. 19. Polar Plot of the Received Signal with Most Optimal. OSTBC Combiner Block (Case-4)

\section{CyberPhyscal Home Automation Demonstration}

Fig. 22 shows the MRASM-ROBOT demonstration (use case) for home automation and security control using the MK1000 controller. The different input interfaces for the access layer can be distinctly seen in Fig. 22. For the input/ output Interface, the motion-controlled light is shown inside the simulated house. On sensing entrance, LDR inside the house checks for the ambient light. If it is not enough, the light comes on. Also, the counter is incremented, and the LCDs the number of persons inside the house. The purpose of the counter is to know when the room is no longer occupied so that the light will be turned off. Also, the Smoke occurrence scenario is captured while the simulated air conditioner is shown inside the system. In this case, the preset temperature is $22^{\circ} \mathrm{C}$. Once the temperature reaches $22^{\circ} \mathrm{C}$, the controller displays a high temperature and the air conditioner comes on. The temperature of the room is maintained at $22^{\circ} \mathrm{C}$ which can run in concurrent IoT Fog designs in complex environments [71], [72].

In Fig. 22, the output of the various neuron circuit linking the $1, \ldots . n+1$ input synaptic circuits is demonstrated on the virtual terminal. For a neuron circuitry having $n$ input signal states, this will yield $n+1$ synaptic output circuits corresponding to the input signal states. The various light conditions are monitored and controlled by the synaptic circuits. At the production settings, the smart home security module is implemented with a secured message digest 5 (MD5) password lock. If the input password is wrong on three trials, the controller will sound an alarm and the IoT module will send the message "security threat" to a dedicated number. In terms of the control system, the main control houses the RF triggered control system. It acts as a link between the input interface, control algorithm, and output interfaces. To verify, the design, different test plans were used and each sub-unit was tested before testing the entire system for validations. A temperature of $22^{\circ} \mathrm{C}$ was injected into the system while the module output changes from zero to 1 .

So far, the proposed MRASM-ROBOT uses Cyberphysical attributes such as diversity to propagate the process variables. Considering the low range coverage distance in Table IV, the proposed system offers relatively better BER, SNR, and high reconfigurability compared with existing works that leveraged OSTBC and HRSM-STBC. This is very significant in IoT-powered neuromorphic robots. The CWDSPT signaling can then fix the complexity error reduction for telemetry data decoding.

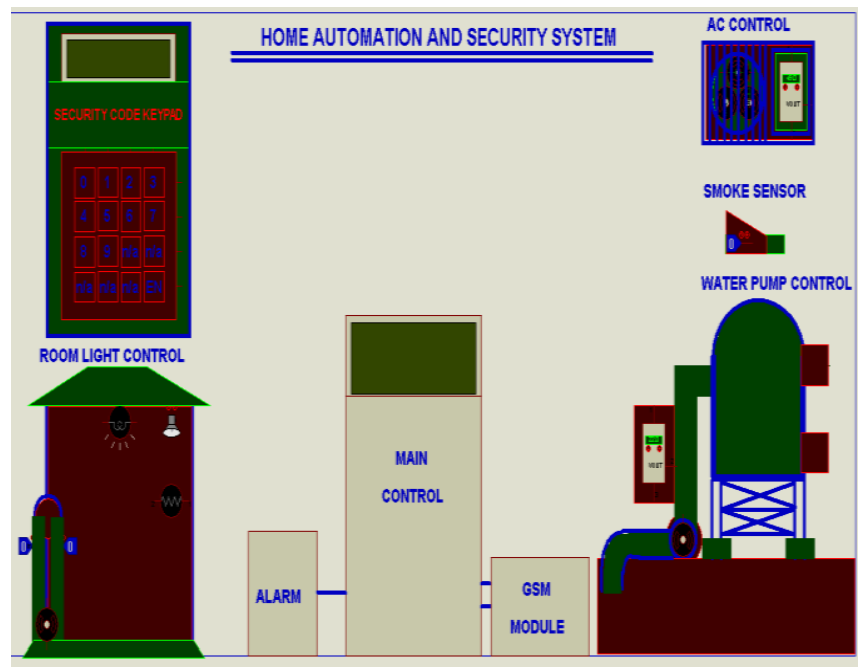

Fig. 20. MRASM-ROBOT (OFF Mode Scenario).

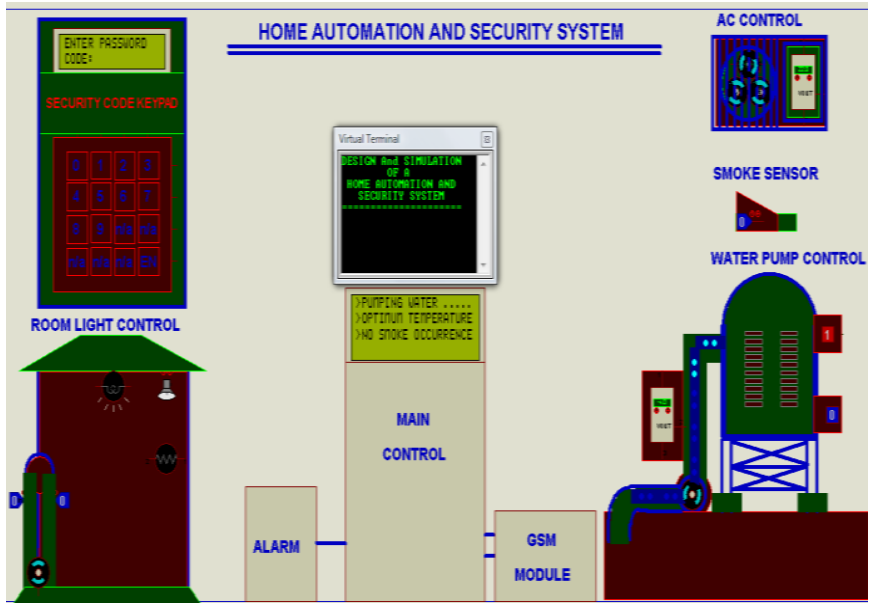

Fig. 21. MRASM-ROBOT (ON Mode Scenario).

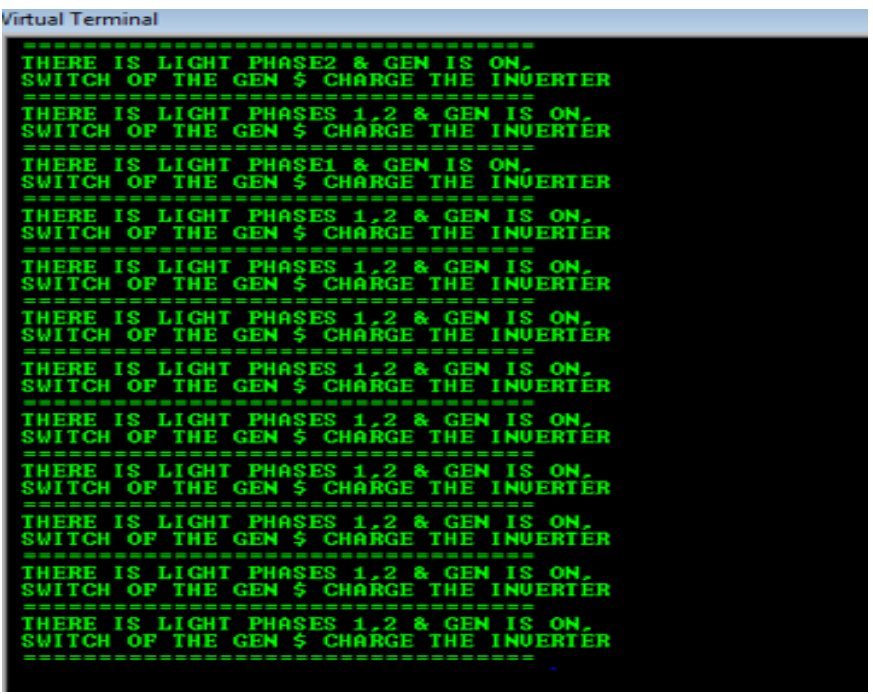

Fig. 22. MRASM-ROBOT Neuron Circuit Control. 
TABLE IV. DIVERSITY COMPARISON

\begin{tabular}{|l|l|l|l|}
\hline Schemes & BER & SNR & Reconfigurability \\
\hline HRSM-STBC [21] & $10^{-5}$ & $5.7 \mathrm{~dB}$ & High \\
\hline OSTBC [66] & $10^{-10}$ & $15 \mathrm{~dB}$ & Moderate \\
\hline MRASM-ROBOT & $9^{-2}$ & $4 \mathrm{~dB}$ & Very High \\
\hline
\end{tabular}

\section{CONCLUSION}

This paper has presented MRASM-ROBOT as a smart home automation system for both security challenged and process-driven environments. The reconfigurable memristive control strategy was used in the synaptic schematics. Dynamic stability with an error-free feedback control loop was realized for design architecture. The CWDSPT was introduced and tested with the OSTBC-CB space diversity scheme. For the process variables, control signals are transmitted with unmodulated high-power continuous wave (CW) for interference minimization. The design offered lower bit error rates leading to minimal error deviation for short distances.

The integration of IoT transmitter and receiver circuit for the device-to-device communication was implemented considering space diversity link reliability. Hence, CWDSPT signaling was optimized for telemetry data decoding within the deployment domain. The work showed the optimization polar plots with the OSTBC-CB for memory stabilization.

Future work will focus on the use of FPGA, containerization, machine learning, and cloud provisioning to address massive scalability concerns under RF antenna diversity. Also, process variable automation such as light systems, air conditioners, overhead tanks, security doors, among others, will be managed with containers.

\section{REFERENCES}

[1] Y. Lv, Y. Fang, W. Chi, G. Chen, and L. Sun, "Object Detection for Sweeping Robots in Home Scenes (ODSR-IHS): A Novel Benchmark Dataset," IEEE Access, vol. 9, pp. 17820-17828, 2021.

[2] S. H. M. S. Andrade, G. O. Content, L. B. Rodrigues, L. X. Lima, N. L. Vijaykumar and C. R. L. Francês, "A Smart Home Architecture for Smart Energy Consumption in a Residence With Multiple Users," IEEE Access, vol. 9, pp. 16807-16824, 2021.

[3] M. -C. Su, J. -H. Chen, A. M. Arifai, S. -Y. Tsai and H. -H. Wei, "Smart Living: An Interactive Control System for Household Appliances," IEEE Access, vol. 9, pp. 14897-14904, 2021.

[4] R. Heartfield, G. Loukas, A. Bezemskij and E. Panaousis, "SelfConfigurable Cyber-Physical Intrusion Detection for Smart Homes Using Reinforcement Learning," IEEE Trans on Information Forensics and Sec., vol. 16, pp. 1720-1735, 2021.

[5] D. Riboni and F. Murru, "Unsupervised Recognition of Multi-Resident Activities in Smart-Homes," IEEE Access, vol. 8, pp. 201985-201994, 2020.

[6] J. Saunders, D. S. Syrdal, K. L. Koay, N. Burke and K. Dautenhahn," Teach Me-Show Me"-End-User Personalization of a Smart Home and Companion Robot," IEEE Trans. on Human-Machine Systems, 46(1), pp. 27-40, 2016.

[7] Y. Zhang and Z. Zeng, "A Multi-functional Memristive Pavlov Associative Memory Circuit Based on Neural Mechanisms," in IEEE Transactions on Biomedical Circuits and Systems, Pg 1-1, Early Access. doi: 10.1109/TBCAS.2021.3108354.

[8] S. Vahdat, M. Kamal, A. Afzali-Kusha and M. Pedram, "Reliability Enhancement of Inverter-Based Memristor Crossbar Neural Networks Using Mathematical Analysis of Circuit Non-Idealities," in IEEE Transactions on Circuits and Systems I: Regular Papers, vol. 68, no. 10, pp. 4310-4323, Oct. 2021, doi: 10.1109/TCSI.2021.3105043.
[9] Y. Song, Q. Wu, X. Wang, C. Wang and X. Miao, "Two MemristorsBased XOR Logic Demonstrated With Encryption/Decryption," in IEEE Electron Device Letters, 42(9), pp. 1398-1401, Sept. 2021, doi: 10.1109/LED.2021.3102678.

[10] X. Xu et al., "MDA: A Reconfigurable Memristor-Based Distance Accelerator for Time Series Mining on Data Centers," in IEEE Trans. on Computer-Aided Design of Integrated Circuits and Sys, 38(5), pp. 785797, 2019, doi: 10.1109/TCAD.2018.2834431.

[11] M. Nourazar, V. Rashtchi, A. Azarpeyvand and F. Merrikh-Bayat, "Code Acceleration Using Memristor-Based Approximate Matrix Multiplier: Application to Convolutional Neural Networks," in IEEE Transactions on Very Large Scale Integration (VLSI) Systems, 26(12), pp. 2684-2695, Dec. 2018, doi: 10.1109/TVLSI.2018.2837908.

[12] X. Xu et al., "Accelerating Dynamic Time Warping With MemristorBased Customized Fabrics," in IEEE Transactions on Computer-Aided Design of Integrated Circuits and Systems, 37(4), pp. 729-741, April 2018, doi: 10.1109/TCAD.2017.2729344.

[13] L. Yavits, R. Kaplan and R. Ginosar, "GIRAF: General Purpose InStorage Resistive Associative Framework,"33(2), pp. 276-287, 1 Feb. 2022, doi: 10.1109/TPDS.2021.3065448.

[14] M. Edmonds, T. Atahary, S. Douglass and T. Taha, "Hardware Accelerated Semantic Declarative Memory Systems through CUDA and MapReduce," in IEEE Transactions on Parallel and Distributed Systems, 30(3), pp. 601-614, 1 ,2019, doi: 10.1109/TPDS.2018.2866848.

[15] L. Chen, C. Li, X. Wang, and S. Duan, "Associate learning and correcting in a memristive neural network," Neural Comput. Appl., vol. 22, no. 6, pp. 1071-1076, 2013.

[16] L. Yang and Z. Zeng, "A memristor-CMOS hybrid circuit for classical conditioning reflex," in Proc. 18th Int. Conf. Inf. Sci. Technol., 2018, pp. 257-261.

[17] J. Sun, G. Han, Z. Zeng and Y. Wang, "Memristor-Based Neural Network Circuit of Full-Function Pavlov Associative Memory With Time Delay and Variable Learning Rate," in IEEE Transactions on Cybernetics, 50(7), pp.2935-2945, 2020, doi: 10.1109/TCYB.2019.2951520.

[18] M. Ansari et al., "PHAX: Physical Characteristics Aware Ex-Situ Training Framework for Inverter-Based Memristive Neuromorphic Circuits," in IEEE Transactions on Computer-Aided Design of Integrated Circuits and Systems, 37(8), pp. 1602-1613, Aug. 2018, doi: 10.1109/TCAD.2017.2764070.

[19] A. Hamdan, H. Hijazi, L. Ros, C. Siclet and A. Al-Ghouwayel, "Interference Analysis for Multi-Carrier Systems Over Fast-Fading Multipath Channels," IEEE Latin-American Conference on Communications (LATINCOM), 2021, pp. 1-6, doi: 10.1109/LATINCOM53176.2021.9647847.

[20] C. Jeong and S. H. Chae, "Simultaneous Wireless Information and Power Transfer for Multiuser UAV-Enabled IoT Networks," in IEEE Internet of Things Journal, 8(10), pp. 8044-8055, 15, 2021, doi: 10.1109/JIOT.2020.3043210.

[21] X. N. Tran, X. Nguyen, M. Le and V. Ngo, "High-Rate Spatially Modulated Space Time Block Code," in IEEE Communications Letters, 22(12), pp. 2595-2598,2018, doi: 10.1109/LCOMM.2018.2872938.

[22] B. Clerckx, J. Kim, K. W. Choi and D. I. Kim, "Foundations of Wireless Information and Power Transfer: Theory, Prototypes, and Experiments," in Proceedings of the IEEE, vol. 110, no. 1, pp. 8-30, Jan. 2022, doi: 10.1109/JPROC.2021.3132369.

[23] I. A. Hernandez-Robles, X. Gonzalez-Ramirez, N. D. Galan-Hernandez and J. M. Ramirez-Arredondo, "Analysis and Design Tool for Wireless Power Transfer for Multiple Applications Purposes," in IEEE Canadian Journal of Electrical and Computer Engineering, 45(1), pp. 24-30, winter 2022, doi: 10.1109/ICJECE.2021.3099402.

[24] L. Wang, J. Li, H. Chen and Z. Pan, "Radial-Flux Rotational Wireless Power Transfer System With Rotor State Identification," in IEEE Transactions on Power Electronics, 37(5), pp. 6206-6216, May 2022, doi: 10.1109/TPEL.2021.3132702.

[25] J. Zhou, P. Zhang, J. Han, L. Li and Y. Huang, "Metamaterials and Metasurfaces for Wireless Power Transfer and Energy Harvesting," in Proceedings of the IEEE, 110(1), pp. 31-55, 2022, doi: 10.1109/JPROC.2021.3127493.. 
[26] X. Wang, Z. Ning, L. Guo, S. Guo, X. Gao and G. Wang, "Online Learning for Distributed Computation Offloading in Wireless Powered Mobile Edge Computing Networks," in IEEE Transactions on Parallel and Distributed Systems, 33(8), pp. 1841-1855, 1 Aug. 2022, doi: 10.1109/TPDS.2021.3129618.

[27] T. Ma, Y. Wang, X. Hu, D. Zhao, Y. Jiang and C. Jiang, "Periodic Energy Control for Wireless Power Transfer System," in IEEE Transactions on Power Electronics, 37(4), pp. 3775-3780, April 2022, doi: 10.1109/TPEL.2021.3129501.

[28] Z. -R. Xu, Y. -F. Ye, L. -S. Wu and J. -F. Mao, "Microstrip Memristive Switch and Its Applications to RF Devices," 2020 International Conference on Microwave and Millimeter Wave Technology (ICMMT), 2020, pp. 1-3, doi: 10.1109/ICMMT49418.2020.9386898.

[29] S. Yu, A. K. Das and Y. Park, "Comments on "ALAM: Anonymous Lightweight Authentication Mechanism for SDN Enabled Smart Homes"," in IEEE Access, vol. 9, pp. 49154-49159, 2021.

[30] M. Ansari et al., "PHAX: Physical Characteristics Aware Ex-Situ Training Framework for Inverter-Based Memristive Neuromorphic Circuits," in IEEE Transactions on Computer-Aided Design of Integrated Circuits and Systems, 37(8), pp. 1602-1613, 2018, doi: 10.1109/TCAD.2017.2764070.

[31] S. Vahdat, M. Kamal, A. Afzali-Kusha and M. Pedram, "Reliability Enhancement of Inverter-Based Memristor Crossbar Neural Networks Using Mathematical Analysis of Circuit Non-Idealities," in IEEE Transactions on Circuits and Systems I: Regular Papers, 68(10), pp. 4310-4323, Oct. 2021, doi: 10.1109/TCSI.2021.3105043.

[32] Z. Fahimi, M. R. Mahmoodi, M. Klachko, H. Nili and D. B. Strukov, "The Impact of Device Uniformity on Functionality of Analog Passively-Integrated Memristive Circuits," in IEEE Transactions on Circuits and Systems I: Regular Papers, 68(10), pp. 4090-4101, 2021, doi: 10.1109/TCSI.2021.3097282.

[33] M. I. Khan, S. Ali, A. A. Ikram and A. Bermak, "Optimization of Memristive Crossbar Array for Physical Unclonable Function Applications," in IEEE Access, vol. 9, pp. 84480-84489, 2021, doi: 10.1109/ACCESS.2021.3087810.

[34] T. Titirsha et al., "Endurance-Aware Mapping of Spiking Neural Networks to Neuromorphic Hardware," in IEEE Transactions on Parallel and Distributed Systems, 33( 2), pp. 288-301, 1 2022, doi: 10.1109/TPDS.2021.3065591.

[35] Y. Zhang and Z. Zeng, "A Multi-functional Memristive Pavlov Associative Memory Circuit Based on Neural Mechanisms," in IEEE Transactions on Biomedical Circuits and Systems, 15(5), pp. 978-993, 2021, doi: 10.1109/TBCAS.2021.3108354.

[36] L. Wang, H. Li, S. Duan, T. Huang, and H. Wang, "Pavlov associative memory in a memristive neural network and its circuit implementation," Neurocomputing, vol. 171, pp. 23-29, Jan. 2016.

[37] Y. Zhou, X. Hu, L. Wang, G. Zhou and S. Duan, "QuantBayes: Weight Optimization for Memristive Neural Networks via Quantization-Aware Bayesian Inference," in IEEE Transactions on Circuits and Systems I: Regular Papers, 68,(12), pp.4851-4861, 2021, doi: 10.1109/TCSI.2021.3115787.

[38] D. Liang, R. Kreiser, C. Nielsen, N. Qiao, Y. Sandamirskaya and G. Indiveri, "Neural State Machines for Robust Learning and Control of Neuromorphic Agents," in IEEE Journal on Emerging and Selected Topics in Circuits and Systems, 9(4), pp. 679-689, 2019, doi: 10.1109/JETCAS.2019.2951442.

[39] S. Roy, A. Banerjee and A. Basu, "Liquid State Machine With Dendritically Enhanced Readout for Low-Power, Neuromorphic VLSI Implementations," in IEEE Transactions on Biomedical Circuits and Systems, 8,(5), pp. 681-695,2014, doi: 10.1109/TBCAS.2014.2362969.

[40] D. Liang and G. Indiveri, "A Neuromorphic Computational Primitive for Robust Context-Dependent Decision Making and Context-Dependent Stochastic Computation," in IEEE Transactions on Circuits and Systems II: Express Briefs, 66,(5), pp. 843-847, 2019, doi: 10.1109/TCSII.2019.2907848.

[41] L. Wang, H. Li, S. Duan, T. Huang, and H. Wang, "Pavlov associative memory in a memristive neural network and its circuit implementation," Neurocomputing, vol. 171, pp. 23-29, Jan. 2016.
[42] Y. Wang, W. Fei, and H. Yu, "SPICE simulator for hybrid CMOS memristor circuit and system," in Proc. Cellular Nanoscale Netw. Appl., 2012, pp. 1-6.

[43] C. Mohan, L. A. Camuñas-Mesa, J. M. De La Rosa, E. Vianello, T. Serrano-Gotarredona and B. Linares-Barranco, "Neuromorphic LowPower Inference on Memristive Crossbars With On-Chip Offset Calibration," in IEEE Access, vol. 9, pp. 38043-38061, 2021, doi: 10.1109/ACCESS.2021.3063437.

[44] L. Yang, Z. Zeng and Y. Huang, "An Associative-Memory-Based Reconfigurable Memristive Neuromorphic System With Synchronous Weight Training," in IEEE Trans on Cognitive and Developmental Syst, 12(3), pp. 529-540, 2020, doi: 10.1109/TCDS.2019.2932179.

[45] 41H. An, Q. An and Y. Yi, "Realizing Behavior Level Associative Memory Learning Through Three-Dimensional Memristor-Based Neuromorphic Circuits," in IEEE Transactions on Emerging Topics in Computational Intelligence, 5(4), pp. 668-678, 2021. doi: 10.1109/TETCI.2019.2921787.

[46] 42M. Payvand, M. E. Fouda, F. Kurdahi, A. M. Eltawil and E. O. Neftci, "On-Chip Error-Triggered Learning of Multi-Layer Memristive Spiking Neural Networks," in IEEE Journal on Emerging and Selected Topics in Circuits and Systems, 10(4), pp. 522-535, 2020, doi: 10.1109/JETCAS.2020.3040248.

[47] 43K. Bai, Q. An, L. Liu and Y. Yi, "A Training-Efficient HybridStructured Deep Neural Network With Reconfigurable Memristive Synapses," in IEEE Transactions on Very Large Scale Integration (VLSI) Systems, 28(1), pp. 62-75, Jan. 2020, doi: 10.1109/TVLSI.2019.2942267.

[48] B. Setz, S. Graef, D. Ivanova, A. Tiessen and M. Aiello, "A Comparison of Open-Source Home Automation Systems," in IEEE Access, vol. 9, pp. 167332-167352, 2021, doi: 10.1109/ACCESS.2021.3136025.

[49] P. Franco, J. M. Martínez, Y. -C. Kim and M. A. Ahmed, "A Framework for IoT Based Appliance Recognition in Smart Homes," in IEEE Access, vol. 9, pp. 133940-133960, 2021, doi: 10.1109/ACCESS.2021.3116148.

[50] S. Yu, N. Jho and Y. Park, "Lightweight Three-Factor-Based PrivacyPreserving Authentication Scheme for IoT-Enabled Smart Homes," in IEEE Access, vol. 9, pp. 126186-126197, 2021, doi: 10.1109/ACCESS.2021.3111443.

[51] M. J. Iqbal et al., "Smart Home Automation Using Intelligent Electricity Dispatch," in IEEE Access, vol. 9, pp. 118077-118086, 2021, doi: 10.1109/ACCESS.2021.3106541.

[52] X. Ran and S. Leng, "Enhanced Robust Index Model for Load Scheduling of a Home Energy Local Network With a Load Shifting Strategy," in IEEE Access, vol. 7, pp. 19943-19953, 2019, doi: 10.1109/ACCESS.2018.2889762.

[53] D. Lan, Z. Pang, C. Fischione, Y. Liu, A. Taherkordi and F. Eliassen, "Latency Analysis of Wireless Networks for Proximity Services in Smart Home and Building Automation: The Case of Thread," in IEEE Access, vol. 7, pp. 4856-4867, 2019, doi: 10.1109/ACCESS.2018.2888939.

[54] R. Zhang et al., "An EOG-Based Human-Machine Interface to Control a Smart Home Environment for Patients With Severe Spinal Cord Injuries," in IEEE Transactions on Biomedical Engineering, vol. 66, no. 1, pp. 89-100, 2019, doi: 10.1109/TBME.2018.2834555.

[55] P. N. Dawadi, D. J. Cook and M. Schmitter-Edgecombe, "Automated Cognitive Health Assessment Using Smart Home Monitoring of Complex Tasks," in IEEE Transactions on Systems, Man, and Cybernetics: Systems, 43(6), pp. 1302-1313, 2013, doi: 10.1109/TSMC.2013.2252338.

[56] S. D. T. Kelly, N. K. Suryadevara and S. C. Mukhopadhyay, "Towards the Implementation of IoT for Environmental Condition Monitoring in Homes," in IEEE Sensors Journal, 13(10), pp. 3846-3853, Oct. 2013, doi: 10.1109/JSEN.2013.2263379.

[57] J. -m. Choi, B. -k. Ahn, Y. -s. Cha and T. -y. Kuc, "Remote-controlled Home Robot Server with Zigbee Sensor Network," SICE-ICASE International Joint Conference, 2006, pp. 3739-3743, doi: 10.1109/SICE.2006.315025.

[58] J. Saunders, D. S. Syrdal, K. L. Koay, N. Burke and K. Dautenhahn, "'Teach Me-Show Me"-End-User Personalization of a Smart Home 
and Companion Robot," in IEEE Transactions on Human-Machine Systems, 46(1), pp. 27-40, 2016, doi: 10.1109/THMS.2015.2445105.

[59] U. Kim and J. Kim, "A Stabilized Feedback Episodic Memory (SF-EM) and Home Service Provision Framework for Robot and IoT Collaboration," in IEEE Transactions on Cybernetics, 50(5), pp. 21102123, May 2020, doi: 10.1109/TCYB.2018.2882921.

[60] K. Park, H. Lee, Y. Kim and Z. Z. Bien, "A Steward Robot for HumanFriendly Human-Machine Interaction in a Smart House Environment," in IEEE Transactions on Automation Science and Engineering, 5(1), pp. 21-25, Jan. 2008, doi: 10.1109/TASE.2007.911674.

[61] A. Corrales Paredes, M. Malfaz and M. A. Salichs, "Signage System for the Navigation of Autonomous Robots in Indoor Environments," in IEEE Transactions on Industrial Informatics, 10(1), pp. 680-688, Feb. 2014, doi: 10.1109/TII.2013.2246173.

[62] J. Berrezueta-Guzman, I. Pau, M. -L. Martín-Ruiz and N. MáximoBocanegra, "Smart-Home Environment to Support Homework Activities for Children," in IEEE Access, vol. 8, pp. 160251-160267, 2020, doi: 10.1109/ACCESS.2020.3020734.

[63] C. Messeri, A. Bicchi, A. M. Zanchettin and P. Rocco, "A Dynamic Task Allocation Strategy to Mitigate the Human Physical Fatigue in Collaborative Robotics," in IEEE Robotics and Automation Letters, 7(2), pp. 2178-2185, 2022, doi: 10.1109/LRA.2022.3143520.

[64] H. Tang, A. Wang, F. Xue, J. Yang and Y. Cao, "A Novel Hierarchical Soft Actor-Critic Algorithm for Multi-Logistics Robots Task Allocation," in IEEE Access, vol. 9, pp. 42568-42582, 2021, doi: 10.1109/ACCESS.2021.3062457.

[65] C. Mohan, L. A. Camuñas-Mesa, J. M. De La Rosa, E. Vianello, T. Serrano-Gotarredona and B. Linares-Barranco, "Neuromorphic LowPower Inference on Memristive Crossbars With On-Chip Offset
Calibration," in IEEE Access, vol. 9, pp. 38043-38061, 2021, doi: 10.1109/ACCESS.2021.3063437.

[66] A. M. K., "OSTBC Transmission in Large MIMO Systems," in IEEE Communications Letters, 20(11), pp. 2308-2311, 2016, doi: 10.1109/LCOMM.2016.2597229.

[67] K. Kassan, H. Farès, D. C. Glattli and Y. Louët, "Performance vs. Spectral Properties for Single-Sideband Continuous Phase Modulation," in IEEE Trans on Comm, 69(7), pp. 4402-4416, 2021, doi: 10.1109/TCOMM.2021.3073792.

[68] Y. Sun, "Optimal Parameter Design of Continuous Phase Modulation for Future GNSS Signals," in IEEE Access, vol. 9, pp. 58487-58502, 2021, doi: 10.1109/ACCESS.2021.3073317.

[69] J. Zhao, Y. Zhang, K. Zeng, W. Rhee and Z. Wang, "A 2.4-GHz Crystal-Less GFSK Receiver Using an Auxiliary Multiphase BBPLL for Digital Output Demodulation With Enhanced Frequency Scaling," in IEEE Transactions on Circuits and Systems II: Express Briefs, vol. 68, no.4, pp. 1143-1147, 2021, doi: 10.1109/TCSII.2020.3032149.

[70] R. Ahmad and A. Srivastava, "PAPR Reduction of OFDM Signal Through DFT Precoding and GMSK Pulse Shaping in Indoor VLC," in IEEE Access, vol. 8, pp. 122092-122103, 2020, doi: 10.1109/ACCESS.2020.3006247.

[71] K. C. Okafor; Guinevere, E.C.; Akinyele, O.O, "Hardware Description Language (HDL): An Efficient Approach to Device Independent Designs for VLSI Market Segments", IEEE Int'l Conf Adaptive Science and Technology (ICAST), 2011, Abuja, 24th-26th, 2011. Pp. 262 - 267. DOI: 10.1109/ICASTech.2011.6145181

[72] K. C. Okafor, G.C. Ononiwu, Sam G. V.C Chijindu, C. C. Udeze "Towards Complex Dynamic Fog Network Orchestration Using Embedded Neural Switch", In International Journal of Computers and Applications, (IJCA), UK,. 2021, 43(2), Pp.91-108. 